\title{
Technology Transfer Challenges: A Case Study of User-Centered Design in NASA's Systems Engineering Culture
}

\author{
Jason Quick \\ Triumph Aerospace Systems - Newport News
}

\begin{abstract}
The Upper Stage (US) section of the National Aeronautics and Space Administration's (NASA) Ares I rocket will require internal access platforms for maintenance tasks performed by humans inside the vehicle. Tasks will occur during expensive critical path operations at Kennedy Space Center (KSC) including vehicle stacking and launch preparation activities. Platforms must be translated through a small human access hatch, installed in an enclosed worksite environment, support the weight of ground operators and be removed before flight - and their design must minimize additional vehicle mass at attachment points. This paper describes the application of a user-centered conceptual design process and the unique challenges encountered within NASA's systems engineering culture focused on requirements and "heritage hardware". The NASA design team at Marshall Space Flight Center (MSFC) initiated the user-centered design process by studying heritage internal access kits and proposing new design concepts during brainstorming sessions. Simultaneously, they partnered with the Technology Transfer/Innovative Partnerships Program to research inflatable structures and dynamic scaffolding solutions that could enable ground operator access. While this creative, technology-oriented exploration was encouraged by upper management, some design stakeholders consistently opposed ideas utilizing novel, untested equipment. Subsequent collaboration with an engineering consulting firm improved the technical credibility of several options, however, there was continued resistance from team members focused on meeting system requirements with pre-certified hardware. After a six-month idea-generating phase, an intensive six-week effort produced viable design concepts that justified additional vehicle mass while optimizing the human factors of platform installation and use. Although these selected final concepts closely resemble heritage internal access platforms, challenges from the application of the user-centered process provided valuable lessons for improving future collaborative conceptual design efforts.
\end{abstract}

INTRODUCTION

This paper describes a user-centered approach to developing Internal Access (IA) Ground Support Equipment (GSE) for the US of NASA's Ares I rocket and the challenges encountered when incorporating new technologies and concepts in a culture focused on system requirements and heritage hardware.

\section{DESIGN OPPORTUNITY}

The Constellation Program includes development of the Ares I Crew Launch Vehicle (CLV) that will send astronauts to the International Space Station (ISS), back to the moon, and on to Mars. Prior to a launch of the Ares I, ground operator access to flight hardware will be required at several locations inside the rocket. Access to these locations will be provided by IA GSE. The creative challenge of designing this IA GSE is being accomplished by members of the NASA design team in the Logistics Support Infrastructure (LSI) subsystem on the US project, including GSE designers and human factors engineers with industrial design backgrounds. Existing full-scale mockups of human access volumes, called Performance Analysis \& Design Demonstrators (PADDs), are available to facilitate prototyping and testing IA GSE concepts (see Figure 1). Furthermore, collaboration with the Technology Transfer/Innovative Partnerships Program at MSFC was encouraged by the LSI subsystem manager to support the incorporation of new technologies in the Ares I US.

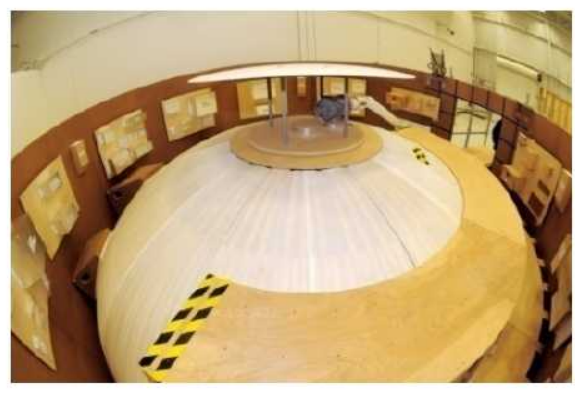

Figure 1: Instrument Unit PADD 


\section{DESIGN CONTEXT}

\section{SYSTEM CONSTRAINTS}

The Ares I system design has functional, physical and operational requirements and constraints. The US segment of the Ares I contains the Instrument Unit (IU) and Interstage volumes that will require internal platforms for ground operators to perform pre-flight launch operations such as maintenance and inspections. The IU is located at the forward end of the US, and the Interstage is located at the aft end. Each cylindrical volume is 18 feet in diameter and has a single 36x36inch access hatch for equipment and ground operators to pass through. A simulated installation of IA GSE through the access hatch is shown in Figure 2.

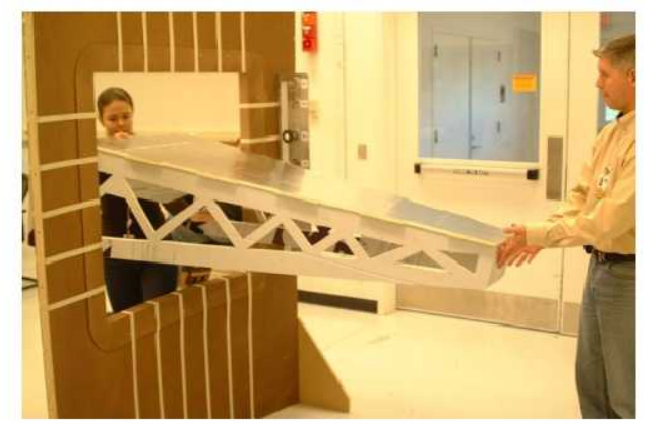

Figure 2: Simulated IA GSE installation

Tasks will occur during critical path operations at Kennedy Space Center (KSC), after the vehicle is stacked and being prepared for launch. The majority of work will take place at the Vehicle Assembly Building (VAB) where 360-degree access will be provided by facility platforms around the outer mold-line of the vehicle. A minimal number of critical, pre-flight tasks will take place on the Mobile Launch Pad (MLP) at the launch site where only limited access will be provided by exterior platforms on access arms. IA GSE must be installed or removed in a single eight-hour work shift to support KSC ground processing timeline requirements.

The IA GSE must meet or support numerous Human Factors, Upper Stage and Constellation requirements, such as the Human Systems Integration Requirements (NASA, 2007). It must be translated through the single $36 \times 36$-inch access hatch and assembled inside the vehicle, as necessary. The resulting work platforms must support the weight of ground operators and their equipment and provide direct access to all components deemed Line Replaceable Units (LRUs) inside the IU and Interstage volumes. The IA GSE must provide fall protection for ground operators and protect the flight hardware from incidental damage caused by Foreign Object Debris (FOD) such as falling tools or equipment. Any platforms, structures and tools must be installed without damage to flight hardware and must be removed before flight. Furthermore, the addition of permanent IA GSE mounting points must be optimized to minimize any unnecessary vehicle mass.

\section{PROJECT CONSTRAINTS}

Project constraints relate to schedules, personnel resources and contractual issues. The primary schedule limitation for initial, viable IA GSE concepts was the Final Layout Reviews (FLORs) for the IU and Interstage structures, which preceded the US Preliminary Design Review (PDR). They provided a chance for incorporating the IA GSE attachment points in the flight hardware drawings and allocating the additional required mass to the overall vehicle mass. The IA GSE concepts required enough detail to justify the added attachment points and could then be refined further after PDR.

During the 6-8 month development period, the GSE design team and Human Factors team could only devote 2-4 engineers full-time to focus on the IA GSE concept development effort. Fortunately, the partnership with Tech Transfer was available for conducting market technology surveys and initiating industry relationships with engineering consulting firms that could support concept development.

\section{CULTURAL CONSTRAINTS}

There have been certain ways of doing things at NASA over the past 50 years. For the Constellation program, NASA has refocused on designing flight hardware, ground facilities, operations and support equipment to lower the costs of maintaining the Ares manned flight capability (NASA, 2008). However, internal access solutions exist for the Space Shuttle components, commercial rockets such as the Delta IV and heritage Saturn vehicles. A common perspective at NASA is that tested, proven hardware should be utilized on subsequent programs. There are several valid reasons for this perspective: extensive requirements and standards are in place that existing hardware meets, concept development requires taking risks on unproven concepts, new hardware certification can be expensive and time-consuming, and changing one part of a complex system design can adversely affect another part of the system. Another important cultural consideration is that KSC ground operations personnel may have the mentality that Ares designers should, "just deliver the vehicle and we will figure it out" with respect to internal access and vehicle maintenance. KSC operators tend to have a "can-do" attitude due to their experience with, and acceptance of, challenging situations where complicated "workaround" strategies may be necessary to complete tasks (Dischinger, 2008).

\section{METHODS}

Development of IA GSE concepts for the US vehicle was carried out by integrating three parallel approaches: user-centered design, technology transfer and systems engineering. They are described here in sequence, but their impacts to the process and project results were interrelated and overlapping. 


\section{USER-CENTERED DESIGN}

The NASA design team at Marshall Space Flight Center (MSFC) applied a user-centered design process by studying heritage internal access kits and generating new concepts focused on providing work platforms that support effective ground operator tasks.

\section{Heritage Access Kits}

An initial study of historical and current equipment designs provided a foundation for further concept development. Engineering illustrations from Saturn-era documentation show internal platform systems used in aft, intermediate and forward sections as well as inside tank volumes (Douglas, 1963). Designs included wallmounted, tank dome-supported and overheadsuspended platform systems. Team members with experience on the Delta IV program provided additional input on wall-mounted, fold-down platforms utilized in human access volumes. Substantial knowledge has also been gained about Space Shuttle operations from engineering walkthroughs of the $\mathrm{VAB}$ and Orbiter Processing Facilities (OPF) at KSC, interactions with KSC ground operators and team-mates on the KSC Ground Operations project. Current internal access kits used for Shuttle processing are similar to platforms used in Saturn vehicles. There are no permanent attachment points provided by the flight vehicle and no platform features designed to accommodate worksite equipment (e.g. lighting, communication, power, tools, and emergency support). On-site modifications to internal access kits are often required (e.g. custom scaffolding, taped-on padding, and special tools).

\section{Concept Development}

Early brainstorming sessions and preliminary research resulted in internal access concepts for further development. During this phase, criticism was minimized and "blue sky" creative thinking was encouraged. The design challenges were reduced into generalized terms and goals, new ideas were proposed during multiple brainstorming sessions and impromptu discussions, and a focus on human interactions and tasks was maintained. Basic web research was conducted to provide examples of existing products and technologies. Early concepts were illustrated with hand sketches, twodimensional (2D) graphics and three-dimensional (3D) computer-aided design (CAD) models. Examples included walking "planks", rail-mounted "trolleys", suspended/rappelling systems, custom scaffolding platforms and inflatable structures. Plans for subsequent market research and concept development through the Technology Transfer group at MSFC were also initiated during this phase.

\section{$\underline{\text { Requirements Generation }}$}

The visualization of initial IA GSE concepts coincided with an initial drafting of design requirements, constraints and goals. Existing requirements for human access and ground operation tasks dictated functional requirements for the IA GSE concepts, such as providing adequate work positions, minimizing risk of damage to flight hardware, meeting operator lifting weight limits and installing a kit in one work shift. Additional system constraints included limits on installation ( $3 \times 3$ foot door), usage at multiple locations (VAB and MLP) and interference with flight hardware keep-out-zones (KOZ). Design goals included permanent attachment points on the vehicle, interfaces for GSE lifting aids, integrated features for operator/vehicle protection and simplified, efficient installation techniques.

\section{Concept Processing}

Upon completion of a six-month period of concept development and requirement generation with multiple sources of creative and technical input, a substantial number of divergent IA GSE system features had been proposed and now required a structured selection process by the core design team. First, each concept that warranted further review was visualized using available and appropriate means: hand sketches, photographs, engineering drawings, 3D CAD models or physical mock-ups. All imagery was displayed in a single room, filling multiple walls and sections of the floor. After brief descriptions and clarifications, team members participated in a qualitative rating session. Each person placed initialed Post-it flags on imagery based on personal impressions: green flags for positive concepts, red flags for negative concepts and blue flags for concepts requiring further investigation. Participants then discussed and defended their ratings, weak concepts were discarded and a subset of strong concepts was selected by the group (see Figure 3 ). This method allowed the team to quickly converge on eighteen concepts. An extensive rating matrix was created to further reduce the number of concepts; however the team realized that several partial concepts could not be validly compared against each other, so the focus was changed to categorization.

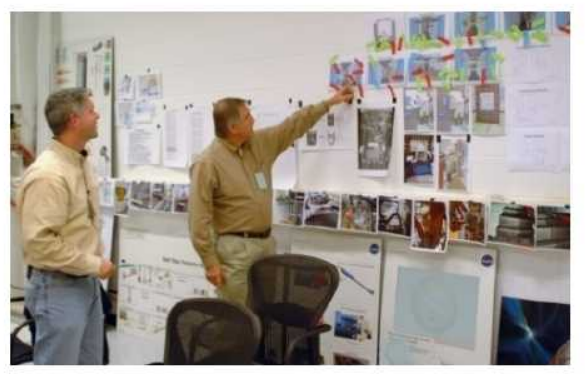

Figure 3: Qualitative concept rating

IA GSE will typically have four basic features: an interface with the flight vehicle, a foundation or structural support, a connection or translation component, and an end element that serves as the human interface. Partial concepts were grouped into the feature categories and combinations were made to create nine complete concepts. The rating matrix was also revised for sharing with the extended design team. 


\section{Stakeholder Involvement}

In order to encourage support for the user-centered design process, the core team presented the process and resulting nine concepts to the extended design team. Comments and criticisms were encouraged during open discussions, and ideas were gathered for modifications to concepts and the rating matrix. For example, due to strong opposition to inflatable structures being the foundation feature of any concept, their use was limited to support during installation. After further discussions, concepts were grouped into eight complete access systems and four "assistive elements". The eight complete systems were also grouped according to the type of operator standing platform: stationary or dynamic (see Figure 4). All twelve concepts were illustrated to a consistent level of detail using $3 D$ CAD software to minimize rating bias.

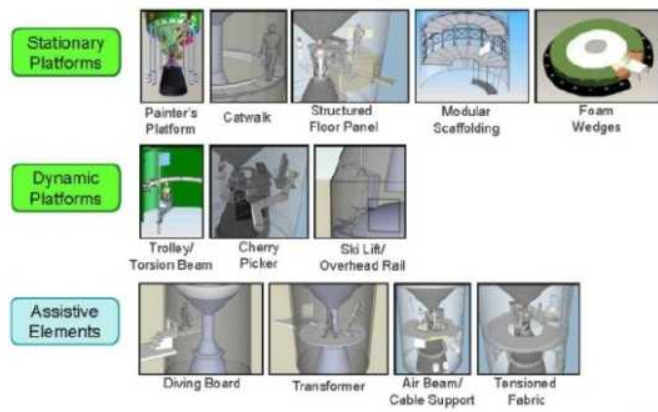

Figure 4: Concept categorization

\section{TECHNOLOGY TRANSFER}

In parallel to the IA GSE design effort, the Human Factors and GSE teams were encouraged by their subsystem manager to utilize the Technology Transfer/Innovative Partnerships Program at MSFC. This group provides focused technology research and facilitates industry partnerships. For the IA GSE team, they searched for companies with unique scaffolding solutions that could meet the design requirements, constraints and goals for the Interstage human access volume. Three companies were profiled and one was selected for further discussions because of its experience with dynamic, moveable scaffolding.

After detailed discussion of the design challenges, the team participated in a short-term engineering consulting contract with the dynamic scaffolding company. Several current IA GSE concepts as well as the complete set of design requirements were shown to the company to encourage new concepts and highlight areas for technical improvement. The company's independentlydeveloped concepts included: inflatable beams, pulleyraised/lowered platforms, suction-cup attachments, torsion beams, robotic "cherry picker" arm and structured floor panels. All concepts were flowed into the team's concept processing phase, previously described.

The Tech Transfer group also searched for companies and researchers developing inflatable products that could be incorporated in IA GSE structures, flooring, assisted lifting or equipment protection. Again, several organizations were profiled and one government researcher was chosen for his subject matter expertise. Current military and commercial applications of inflatable structures showed potential for use in NASA's Ares program. However, due to resource and schedule limitations, as well as opposition among IA GSE stakeholders, the capabilities of inflatable structures was not explored further by the IA GSE design team.

\section{SYSTEMS ENGINEERING}

As the IA GSE concepts were in development, the flight vehicle design was approaching Final Layout Reviews (FLORs), which were followed closely by the US Preliminary Design Review (PDR). These are important system engineering milestones that motivated the IA GSE design team to deliver viable concepts that justified specific requests for flight vehicle interfaces (e.g. permanent mounting locations) and impacts to vehicle mass (NASA, 2007).

The need for IA GSE was established by the requirement for ground operator access inside the US element for nominal pre-launch tasks and off-nominal maintenance tasks. Past and current NASA programs have satisfied this requirement with tested, certified hardware. As part of the LSI subsystem of the US element, any new IA GSE designs must meet numerous system requirements at multiple NASA program levels. Requirement compliance for these hardware designs will be verified by demonstration, analysis, inspection or testing (NASA, 2007).

Proposed IA GSE concepts and the user-centered design process were presented with a systems engineering perspective. Concepts described the context of ground operations at KSC to highlight handling and installation concerns, and technical parameters addressed stakeholder concerns such as structural loading, operator safety and hardware protection. Engineering feasibility studies were conducted with subject matter experts and the concept rating matrix was refined based on key system requirements and input from the extended design team. Twelve stakeholders from various systems integration disciplines submitted ratings to determine the final concept selections.

\section{RESULTS}

Stakeholder voting resulted in separate IA GSE recommendations for the Interstage and the IU. The Interstage concept, shown in Figure 5, consists of structural floor panels installed circumferentially to attachment points on the Interstage inner wall. Small vertical beams allow multiple floor panels to be mounted at selected elevations. Each floor panel will be similarly sized and weighted for easy translation through the Interstage access hatch for installation. The main floorelevation level will have full $360^{\circ}$ access and be the 
staging platform to access other levels within the Interstage (Smith, 2008). The request for permanent attachment points on the Interstage inner wall was accepted by the US PDR review board.

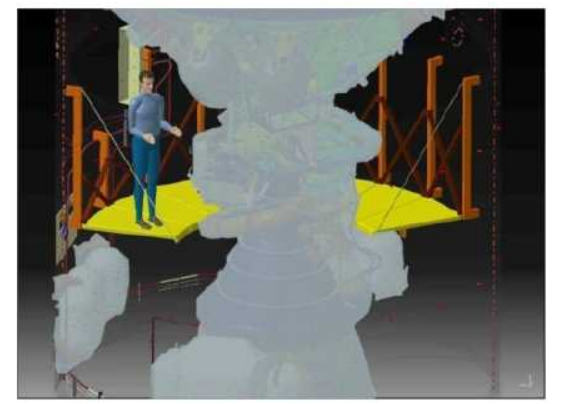

Figure 5: Interstage IA GSE concept

The IA GSE for the IU, shown in Figure 6, consists of two levels of wedge-shaped components that lie circumferentially on top of the Liquid Hydrogen (LH2) tank dome Thermal Protective System (TPS). Each piece will be similarly sized and weighted to allow easy entrance through the IU access hatch for installation. The lower level will slide against the IU inner wall surface and the upper level will rest against the lower level (Smith, 2008).

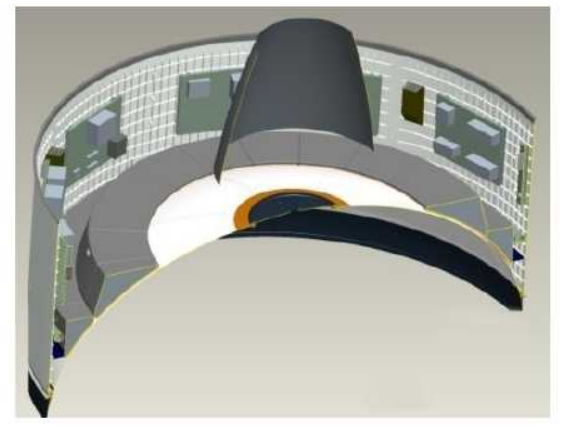

Figure 6: Instrument Unit IA GSE concept

The FLOR and PDR milestones signified that the IA GSE concepts were approved. With Interstage attachment points and IU keep out zones reserved, the design team could progress with detailed IA GSE concepts and mockup development.

\section{DISCUSSION}

\section{ISSUES \& CHALLENGES}

While the exploration of creative, technology-oriented concepts was encouraged by upper management, some IA GSE stakeholders opposed concepts utilizing untested technologies or configurations. Specifically, the use of inflatable products and structures was repeatedly rejected with the rationale that current scaffolding requirements do not sanction their use. Hardware testing timelines and certification costs were also cited as risks to acceptance of IA GSE designs utilizing inflatable structures. Furthermore, concepts featuring moving platforms received low ratings for perceived safety issues related to emergency egress. Concepts that were unfamiliar or did not strictly adhere to system requirements had a hard time gaining support. As a result, the final approved concepts closely resemble existing internal access kits used in current space flight vehicles: wall-mounted, fold-down platforms are used in the Delta IV program and foam wedges are used for human access inside the Space Shuttle external tank structure.

IA GSE design team members disagreed on the appropriate methods for producing concept imagery. In much of the NASA engineering community, hardware is designed using parametric, solid modeling in the $3 \mathrm{D}$ CAD software Pro/ENGINEER. Simulations of human interactions with flight hardware are created in Digital Enterprise Lean Manufacturing Interactive Application (DELMIA) software using models imported from Pro/ENGINEER. However, the engineering consulting company used Google SketchUp software to create realistic 3D models of IA GSE concepts in much less time than is possible in traditional CAD software. During the concept processing phase, team members also used SketchUp to quickly create $3 D$ models of the twelve concepts for voting. Some team members were concerned that images of SketchUp models presented to IA GSE stakeholders could have been misconstrued as developed $C A D$ designs rather than proposed concepts that may not have been scaled to exact vehicle dimensions. These models could have been converted to realistic "CAD-looking" models for official project documentation, but the decision was made to recreate concepts in Pro/ENGINEER. This resulted in significant additional time spent by team members on CAD modeling prior to delivering the final report.

Challenges were also encountered in coordinating the short-term engineering consulting contract. This was the first utilization of the Technology Transfer/Innovative Partnerships Program on the Upper Stage project, and contract officials were careful to avoid situations that could limit future partnerships or larger contract opportunities. Additionally, the IA GSE team could not predict what level of background information was most appropriate to share with the scaffolding company. Some stakeholders felt that NASA-generated concepts shown to the scaffolding company may have negatively impacted the quality of their concepts. Others appreciated that the company added engineering credibility to existing IA GSE concepts.

\section{SUCCESSES}

The primary goal of the IA GSE design team was to provide input to the Interstage and IU designs for IA GSE interfaces. That goal was reached at the US PDR when the vehicle design included rows of permanent attachment points on the Interstage wall and dedicated keep-out zones for foam wedges on the lower portion of the IU inner wall. This required vehicle designers to relocate flight components, purge ducts and power/data lines. 
Potential for efficient human access and maintenance tasks was improved by IA GSE vehicle interfaces being "on the books", and increased awareness of human tasks inside the vehicle among system designers has been noted by members of the Human Factors team. The positive exposure gained by implementing this usercentered design process has increased project acceptance of Human Factors approaches to the integrated system design.

Additionally, risk may have been reduced by rewording a structural requirement levied on the LH2 dome, which now explicitly states that the tank's dome surface in the IU must support human and GSE loading while pressurized.

\section{LESSONS LEARNED}

The challenges and successes experienced throughout the IA GSE design process provided valuable lessons for future concept development efforts:

\section{Focus on system \& mission benefits}

User-centered concepts are viewed most positively when stated in terms of vehicle protection and human safety. Minimal increases to vehicle mass, the ground operations timeline and operating costs are also critical for acceptance - reductions are even better.

\section{$\underline{\text { Understand system requirements \& rationale }}$}

New concept developers must understand the rationale behind system requirements. The modification of existing requirements may be possible if the original intent is satisfied. In the requirements-focused design environment at NASA, it may be more realistic to change requirements than to avoid them altogether.

\section{$\underline{\text { Concept visualization matters }}$}

Communicate visual concepts consistently and at the appropriate level of detail for your current audience. Hand sketches, physical mockups and 3D computer models are effective at different stages of concept development.

\section{Encourage conflict}

Learn to appreciate and encourage disagreement. Stakeholders with strong opposing views may bring valuable experience and perspectives to discussions that can be essential to identifying and resolving technical issues.

\section{Maintain high-level support}

Management support of creative processes is needed to develop an innovative work culture. While motivated individuals and small teams can initiate new practices, high-level support provides a foundation for incorporating new technologies and industry expertise.

\section{CONCLUSION}

Designing space launch systems is challenging work. Complex engineering problems, requirements-focus and resistance to change will continue to influence the NASA design culture's response to unproven concepts. The process of developing IA GSE for the Ares I Upper Stage demonstrated the importance of presenting credible concepts that meet system requirements.

There is considerable opportunity for the NASA design team to refine concept details during the IA GSE mockup development effort. Operator-centered system designs can be guided by current KSC operator insights, component and technology research and industry partnerships. Also, an improved working relationship with the Technology Transfer group can yield a portfolio of innovative technologies and applications. Finally, task simulations utilizing full-scale PADD facilities, physical mockups and human modeling software will continue to benefit collaborative design efforts focused on humansystems integration.

\section{REFERENCES}

Dischinger, H. C. (2008). The first development of human factors engineering requirements for application to ground task design for a NASA flight program. 38th International Conference on Environmental Systems ( $p$. 1). San Francisco, CA: SAE International.

Douglas Aircraft Corporation, Inc. (1963). Model Specification Ground Support Equipment Saturn S-IVB Stage. Santa Monica, CA: Douglas Missile \& Space Systems Division.

National Aeronautics and Space Administration. (2008). Ares I Operational Concepts Document - CxP 72032. Huntsville, AL: George C. Marshall Space Flight Center.

National Aeronautics and Space Administration. (2007). Constellation Program Human-Systems Integration Requirements - Revision A - CxP 70024. Washington, D.C.: National Aeronautics and Space Administration.

National Aeronautics and Space Administration. (2007). NASA Systems Engineering Handbook. Washington, D.C.: NASA Scientific and Technical Information program.

Smith, K. A. (2008). Ares1 Upper Stage (US) Internal Access (IA) Ground Support Equipment (GSE) Concept Development Project - Final Report. Huntsville, AL: Jacobs ESTS Group.

\section{CONTACT}

Jason Quick

EV82/Vehicle Engineering and Integration Division

MSFC, AL 35812

jason.c.quick@nasa.gov 
Jason Quick, Human Factors Engineer Triumph Aerospace System - Newport News Marshall Space Flight Center, Huntsville, AL

International Conference on Environmental Systems July 16, 2009

Technology Transfer Challenges: A Case Study of User-Centered Design in NASA's Systems Engineering Culture

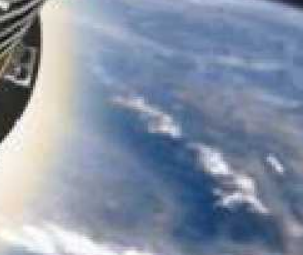

2009-01-2404

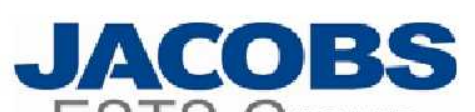


Jason Quick, Human Factors Engineer Triumph Aerospace System - Newport News Marshall Space Flight Center, Huntsville, AL

International Conference on Environmental Systems (ICES) Human Factors for Space Missions: Ground and Flight Operations July 16, 2009 - Savannah, GA

Technology Transfer Challenges: A Case Study of User-Centered Design in NASA's Systems Engineering Culture 


\section{AGENDA}

\section{Design Opportunity}

- Problem statement

- Resources

- Constraints

\section{Concept Development}

- User-centered design

- Technology transfer

- Systems engineering

\section{Results \& Discussion}

- Final concepts

- Challenges \& successes

- Lessons learned 


\section{DESIGN OPPORTUNITY}

\section{Problem Statement:}

$\longleftarrow$ Instrument Unit

Develop internal access Ground Support Equipment (GSE) to support pre-launch ground crew tasks inside Upper Stage volumes of the Ares I Crew Launch Vehicle. 


\section{DESIGN OPPORTUNITY}

$\longleftarrow$ Instrument Unit

$\longleftarrow$ Interstage

\section{Problem Statement:}

Develop internal access Ground Support Equipment (GSE) to support pre-launch ground crew tasks inside Upper Stage volumes of the Ares I Crew Launch Vehicle.

- Kennedy Space Center (KSC) launch site

- Vehicle Assembly Building (VAB)

- Mobile Launch Platform (MLP)

- Human access to components inside vehicle

- Protect ground crew \& hardware 


\section{DESIGN OPPORTUNITY}

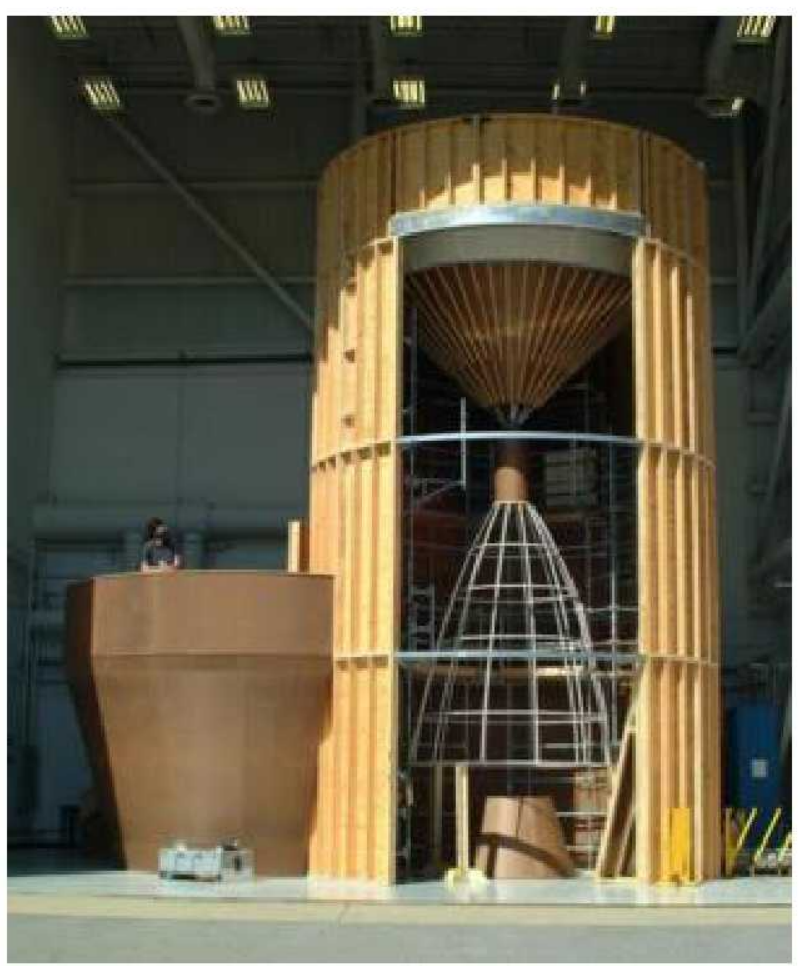

\section{Resources:}

- Creative Human Factors/GSE Design team

- Existing full-scale mockup capabilities

- Collaboration with Technology Transfer

- Feedback from heritage solutions 


\section{AGENDA}

\section{Design Opportunity}

- Problem statement

- Resources

- Constraints

Concept Development

\begin{tabular}{|l|}
\hline - System \\
- Project \\
- Cultural \\
\hline
\end{tabular}

- User-centered design

- Technology transfer

- Systems engineering

Results \& Discussion

- Final concepts

- Challenges \& successes

- Lessons learned 


\section{DESIGN OPPORTUNITY}

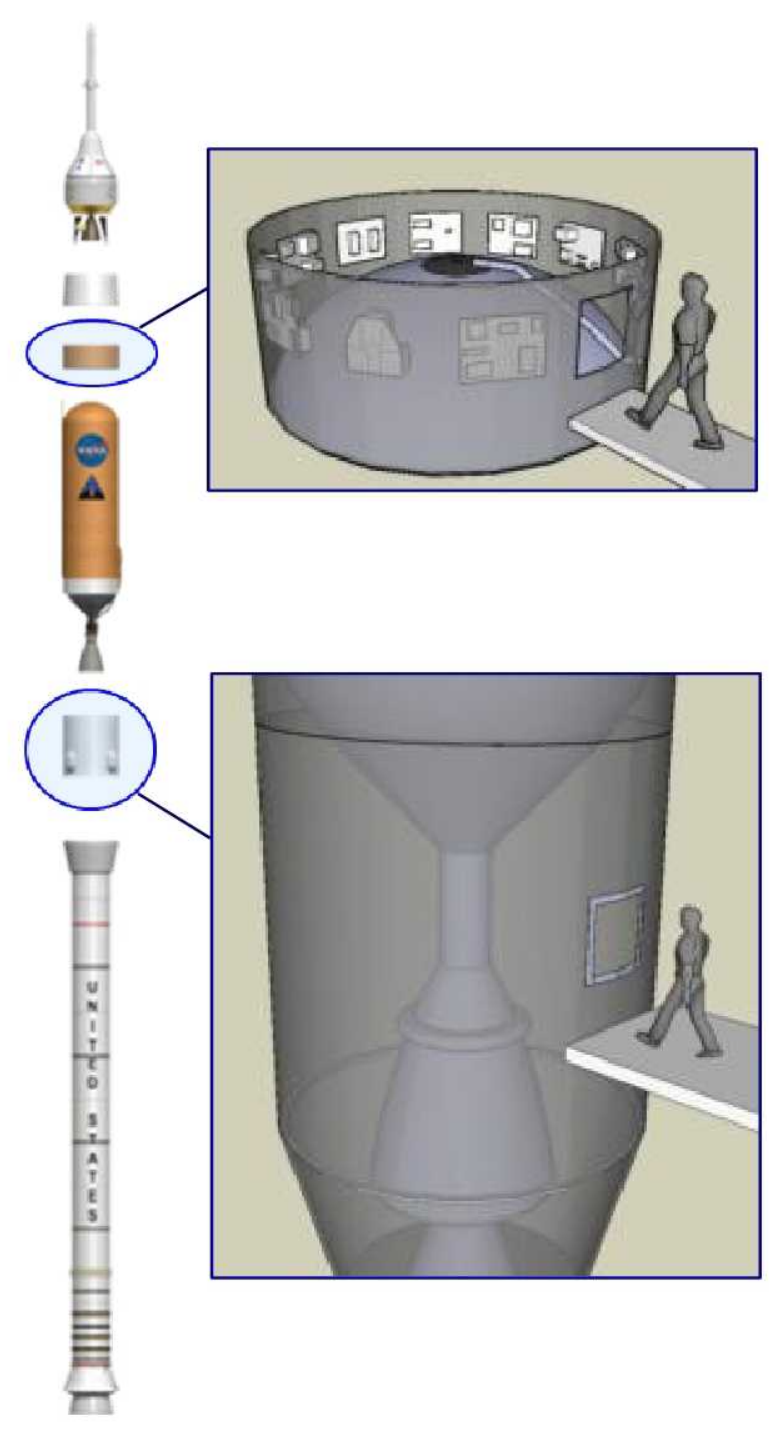

\section{Constraints > System:}

- Current vehicle configuration

- 36"x36" (91.4cm) access door

- Liquid Hydrogen (LH2) dome

- Interstage volume

- Several Keep-Out Zones

- Minimize vehicle mass for access 


\section{DESIGN OPPORTUNITY}

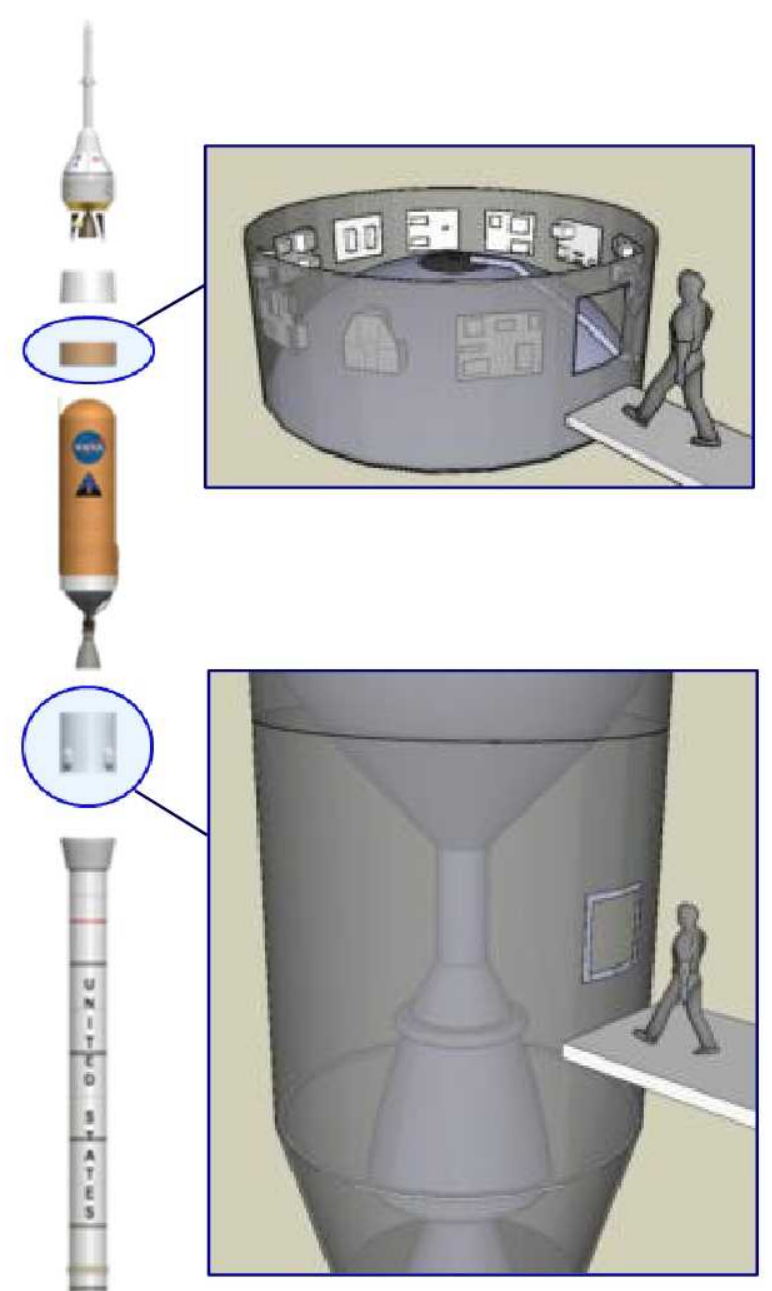

\section{Constraints > System:}

- Current vehicle configuration

- 36"x36" (91.4cm) access door

- Liquid Hydrogen (LH2) dome

- Interstage volume

- Several Keep-Out Zones

- Minimize vehicle mass for access

- Ground operations

- Critical Path timeline constraints

- Stacked vehicle w/ possible sway

- Heavy lift: Line Replaceable Units (LRUs)

- Grasp, lift \& translate

- Platforms removed before flight 


\section{DESIGN OPPORTUNITY}

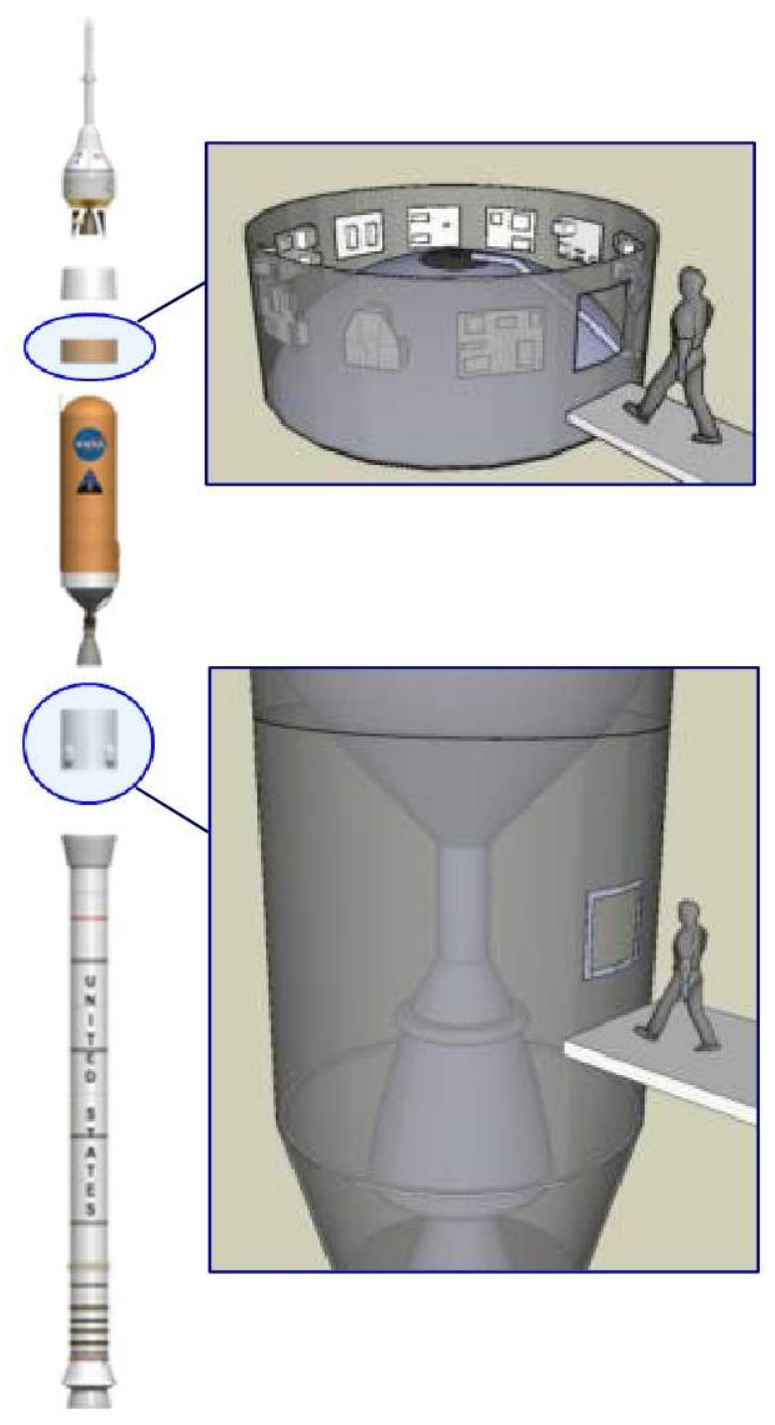

\section{Constraints > System (cont.):}

- Human factors \& design requirements

- Visual \& physical access

- Weight limitations

- Handling complexity

- Lighting

- Safety (egress, etc) 


\section{DESIGN OPPORTUNITY}

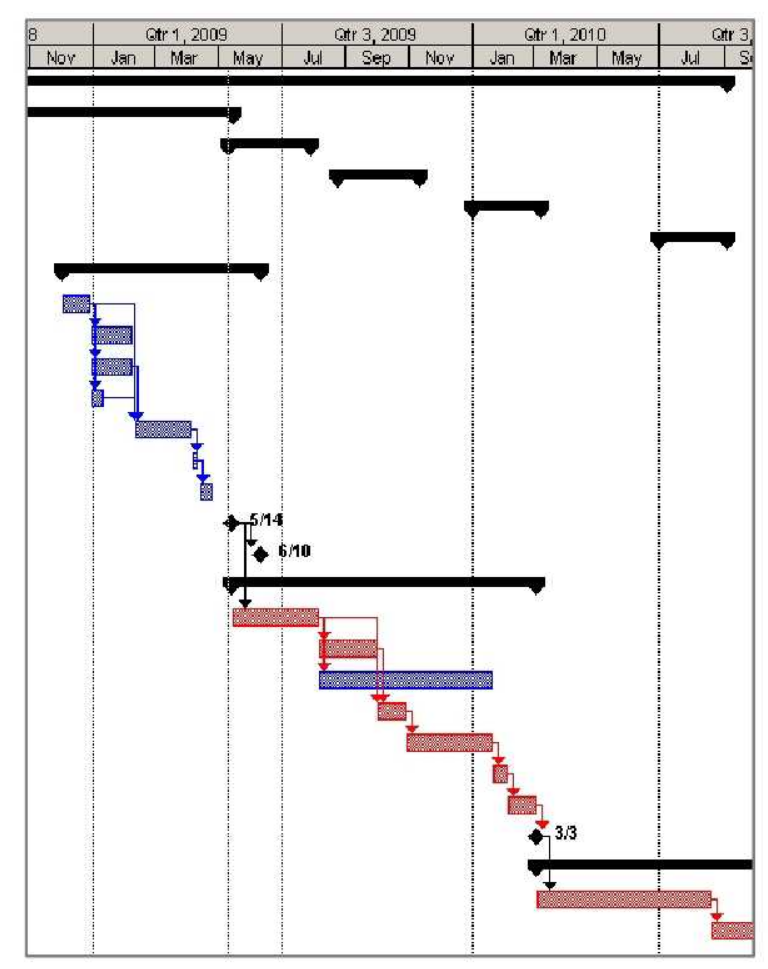

\section{Constraints > Project:}

- Timeline milestones

- Final Layout Reviews (FLORs)

- Preliminary Design Review (PDR)

- Manpower \& facilities

- Technology/material costs 


\section{DESIGN OPPORTUNITY}

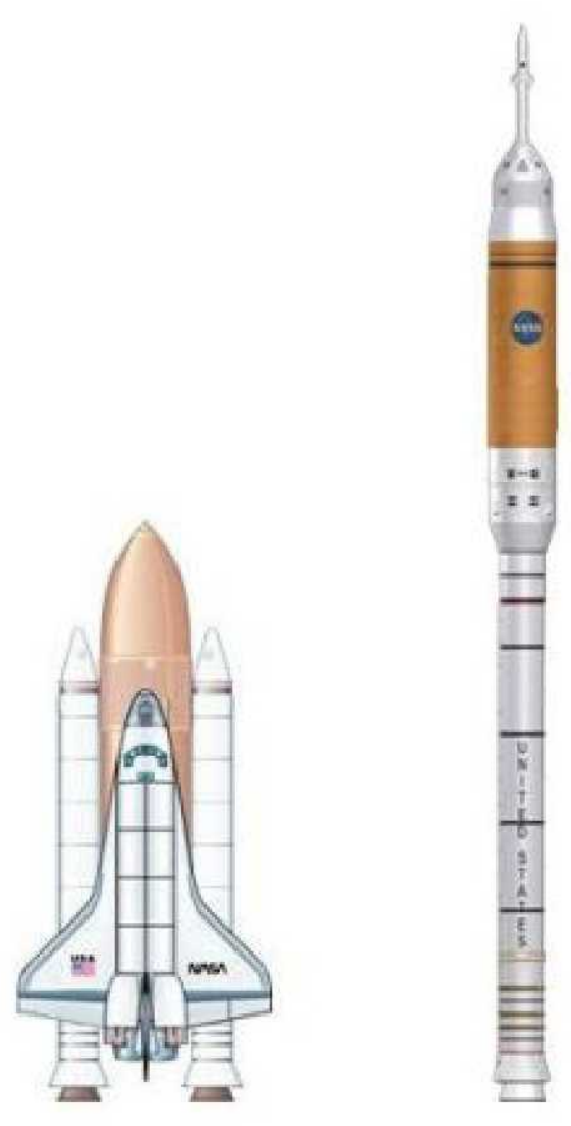

\section{Constraints > Cultural:}

- Certified hardware

- Low tolerance for risk

- Deferring to KSC solutions 


\section{AGENDA}

Design Opportunity

- Problem statement

- Resources

- Requirements \& constraints

\section{Concept Development}

- User-centered design

- Technology transfer

- Systems engineering

\section{Results \& Discussion}

- Final concepts

- Challenges \& successes

- Lessons learned 


\section{CONCEPT DEVELOPMENT}

\section{Internal Access (IA) GSE Concept Development}

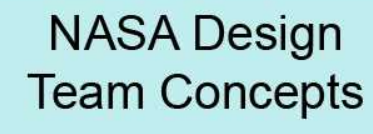

Tech Transfer \& Eng. Consulting

Concept Processing

\& Rating System 


\section{CONCEPT DEVELOPMENT}

\section{User-Centered Design $>$ Brainstorming}
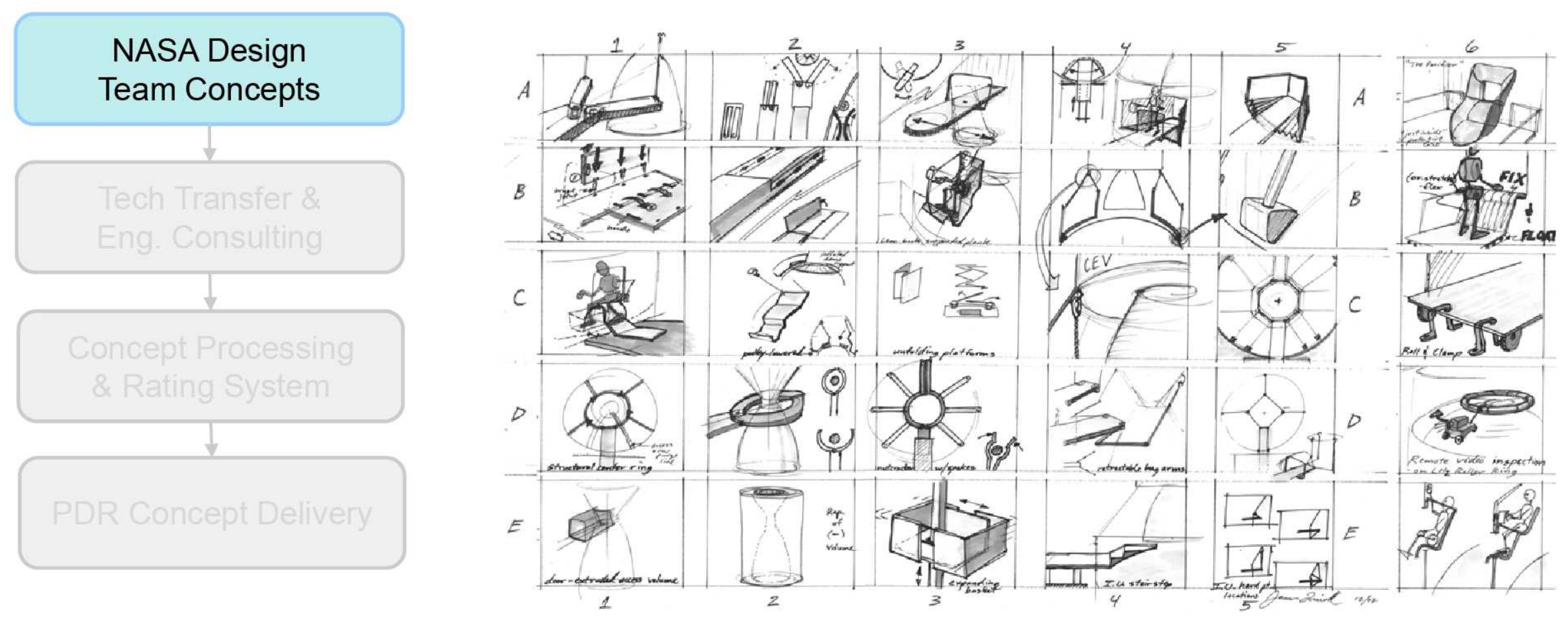


\section{CONCEPT DEVELOPMENT}

\section{User-Centered Design > Idea Development}
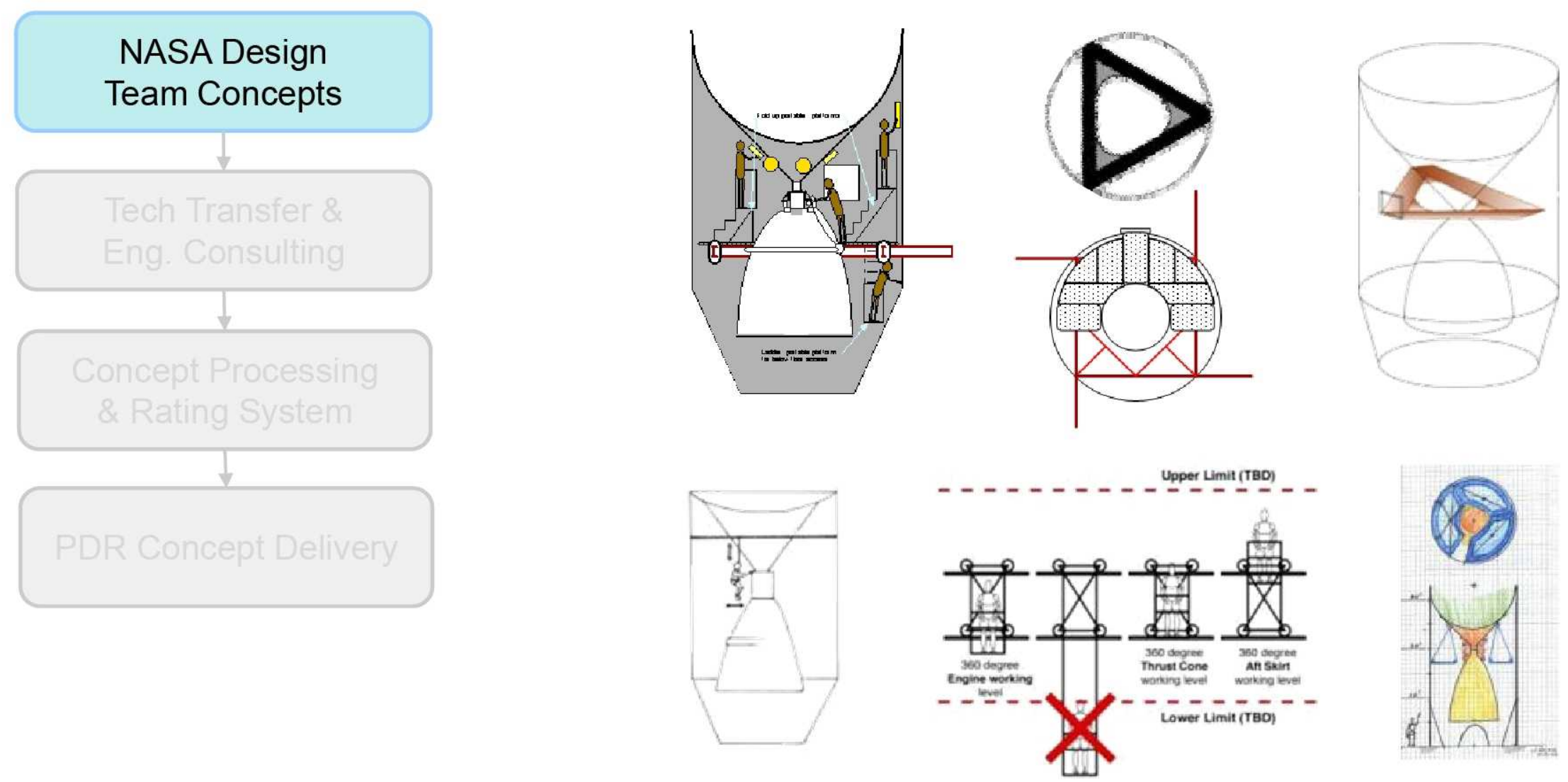


\section{CONCEPT DEVELOPMENT}

\section{User-Centered Design > Physical Mockups}
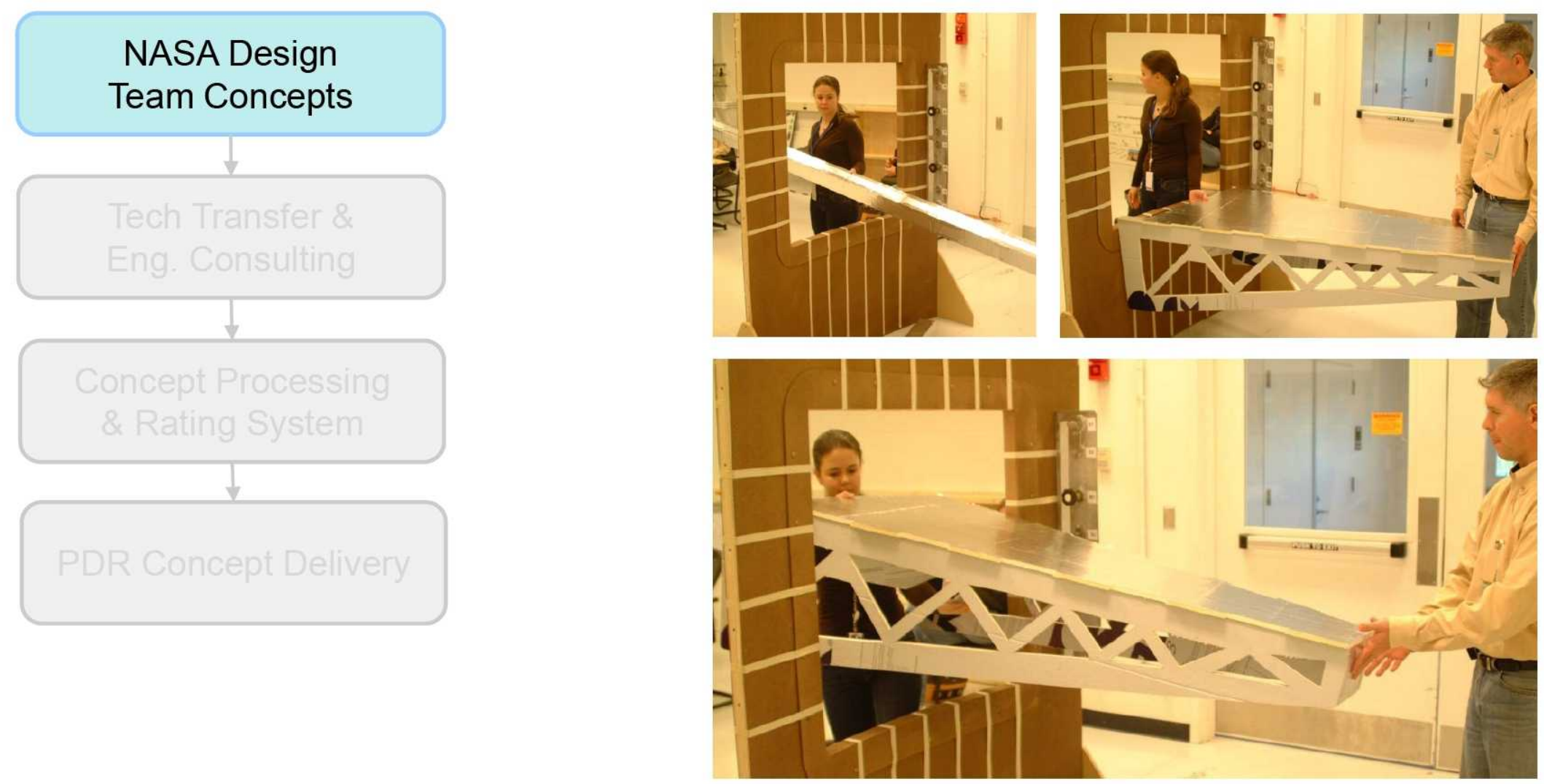


\section{CONCEPT DEVELOPMENT}

\section{Technology Transfer > Industry Research}

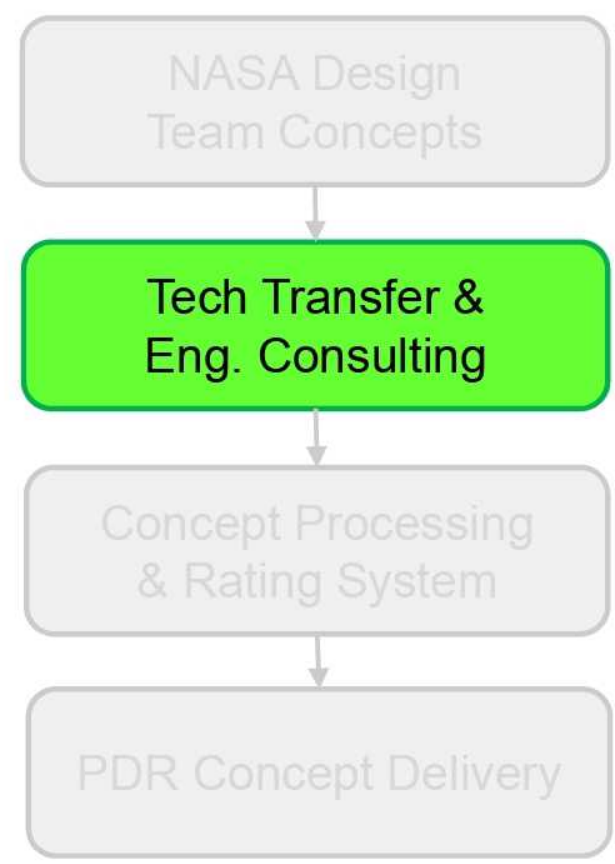

- Custom scaffolding

- Lightweight parts

- Dynamic features

- Inflatable structures

- Military/Naval

- Aerospace

- Commercial 


\section{CONCEPT DEVELOPMENT}

Technology Transfer > Engineering Consulting

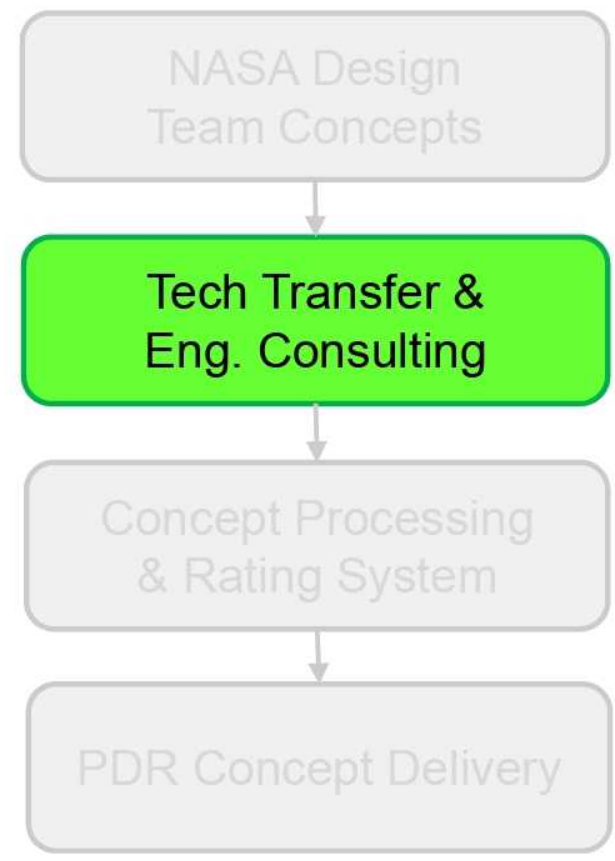

- System design experience

- Robotics, inflatables, fabrics

- Technical feasibility

- Cherry picker foundation

- Structural torsion beam
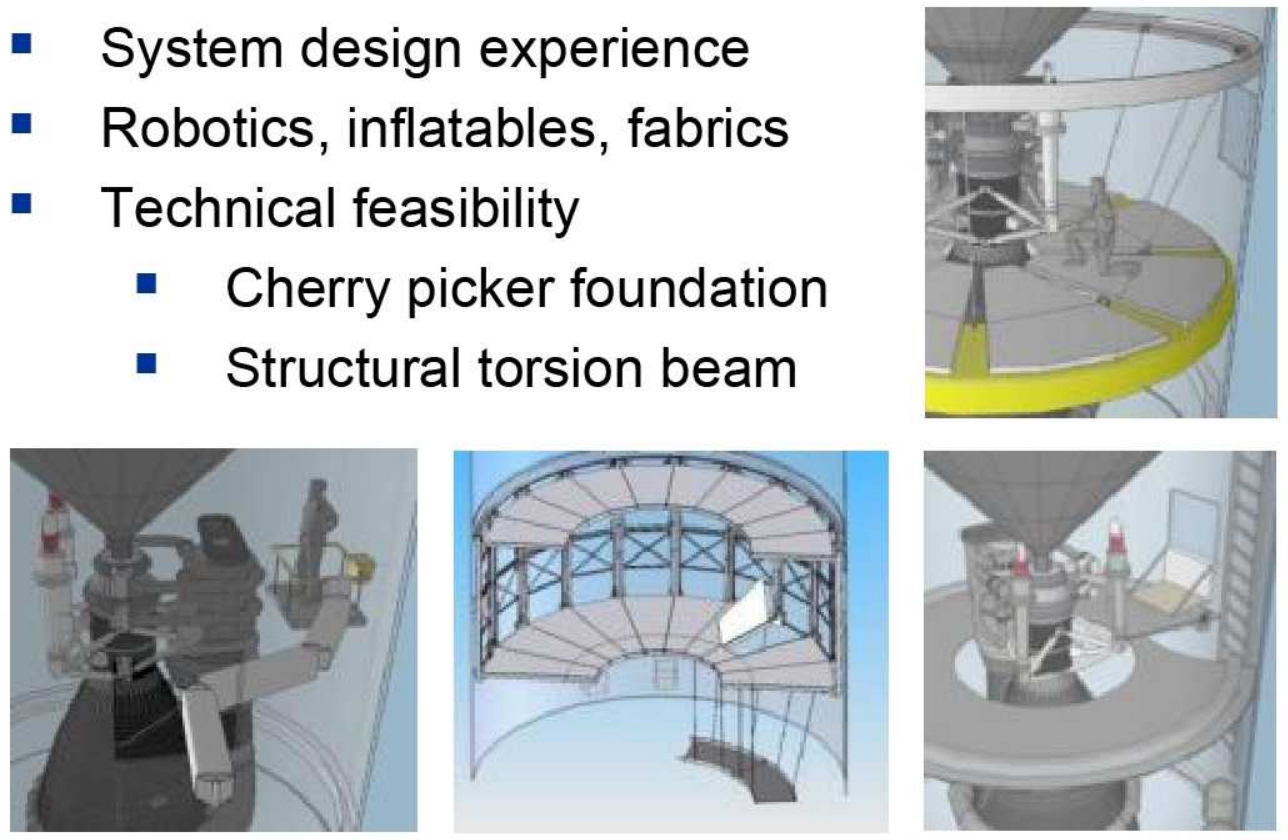


\section{CONCEPT DEVELOPMENT}

\section{Technology Transfer > Engineering Consulting}

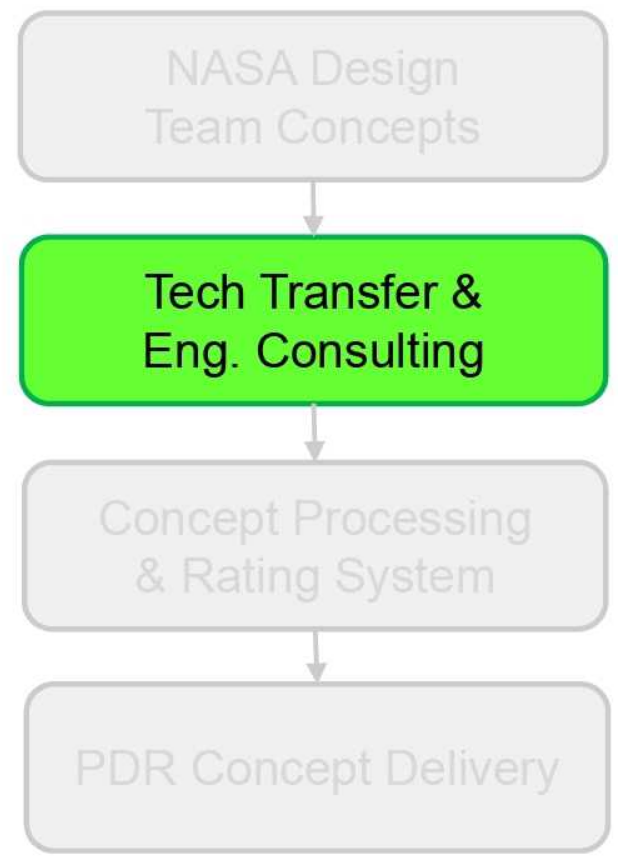

- System design experience

- Robotics, inflatables, fabrics

- Technical feasibility

- Cherry picker foundation

- Structural torsion beam

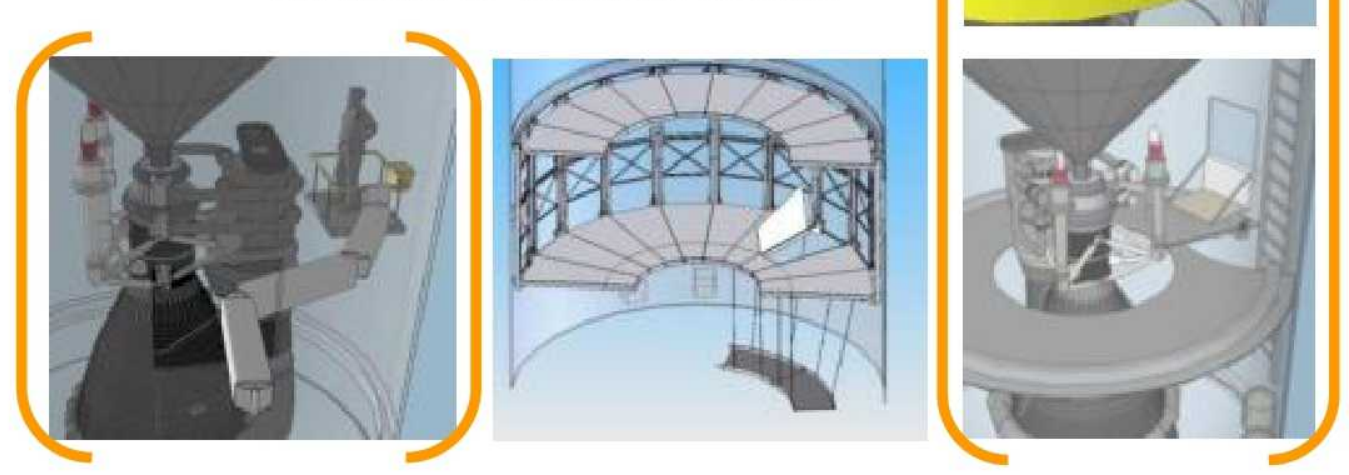




\section{CONCEPT DEVELOPMENT}

\section{Systems Engineering > Concept Processing}
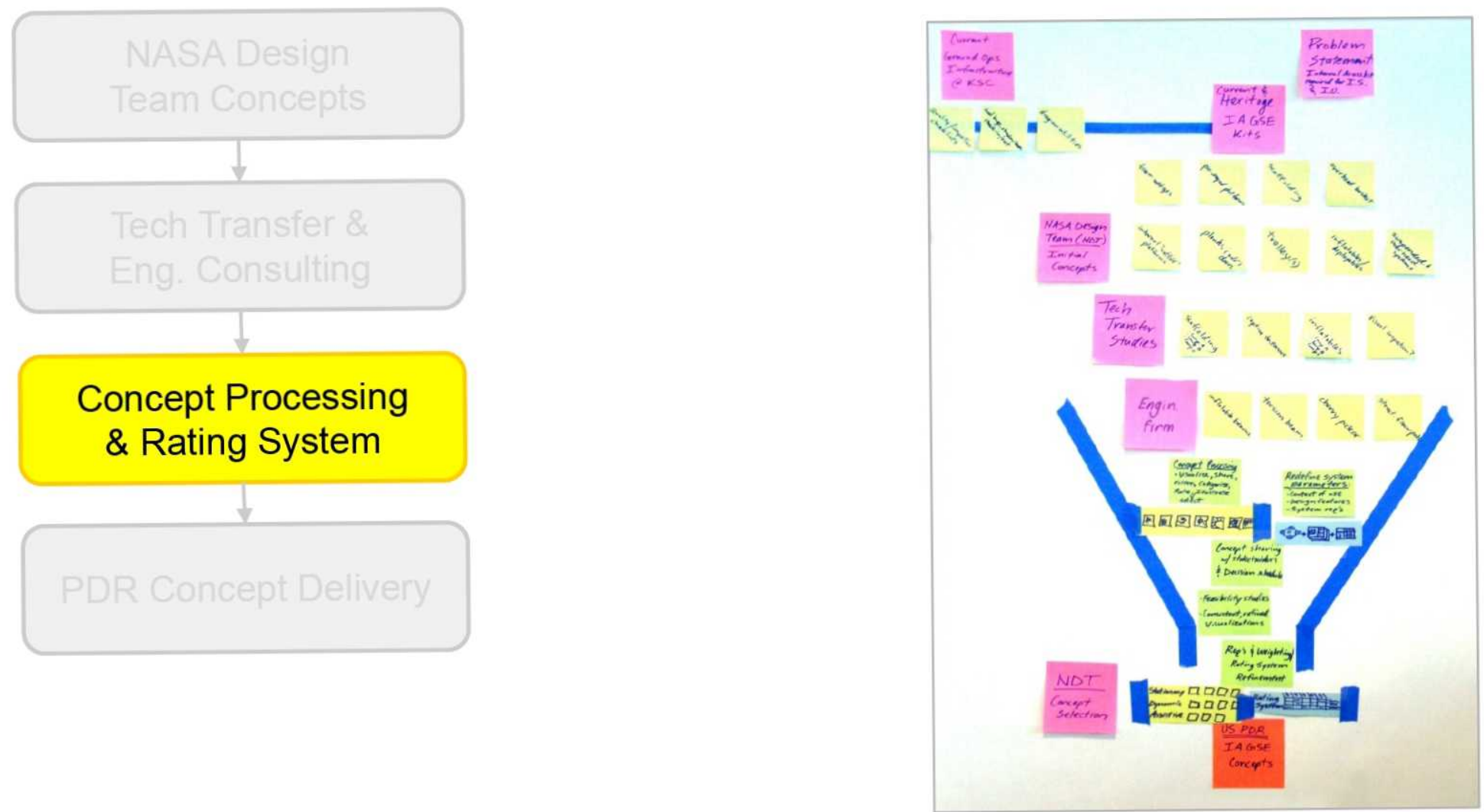


\section{CONCEPT DEVELOPMENT}

\section{Systems Engineering > Initial Rating Criteria}
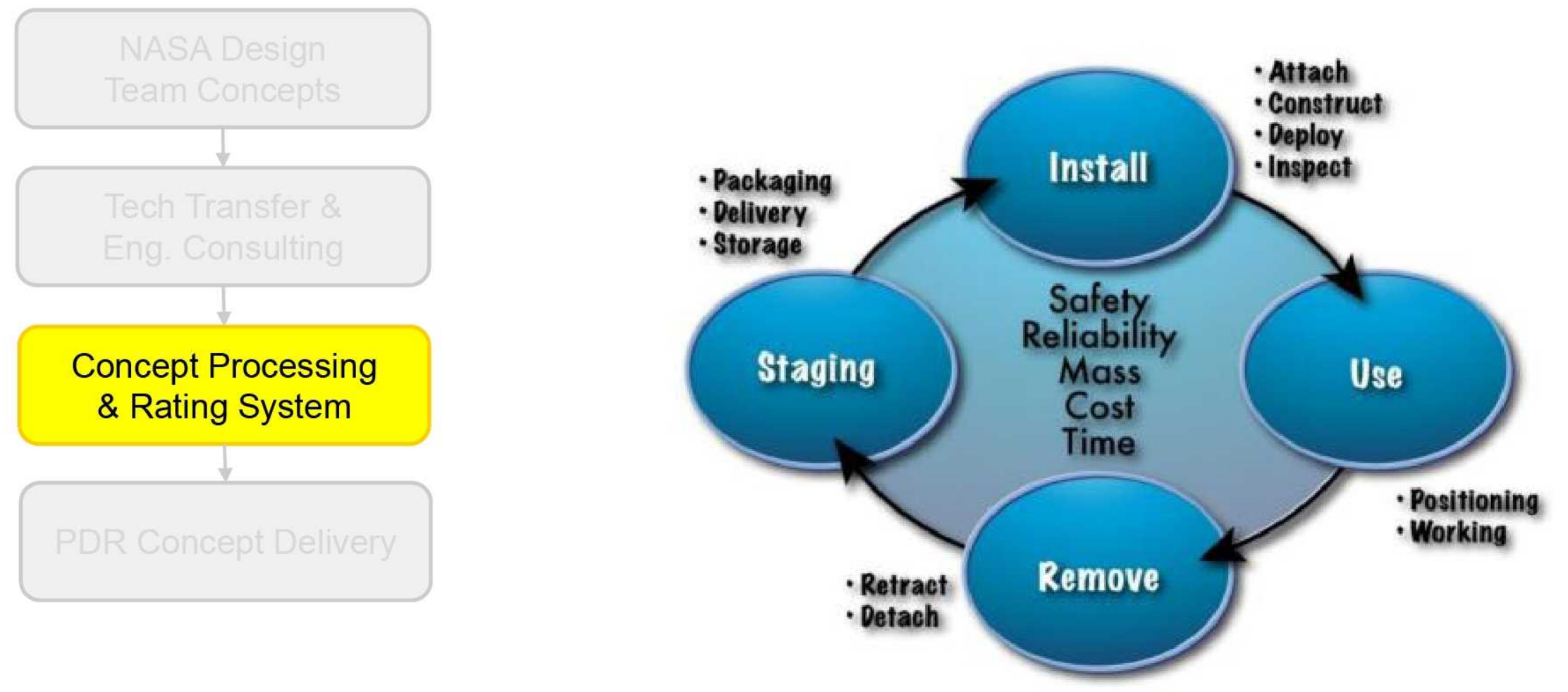


\section{CONCEPT DEVELOPMENT}

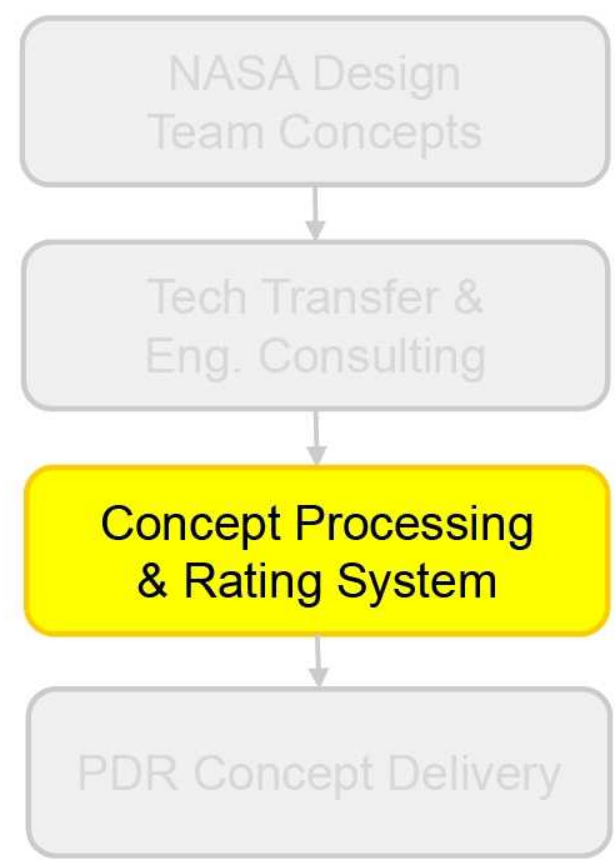

Systems Engineering > Team Sessions for Concept Organizing \& Grouping

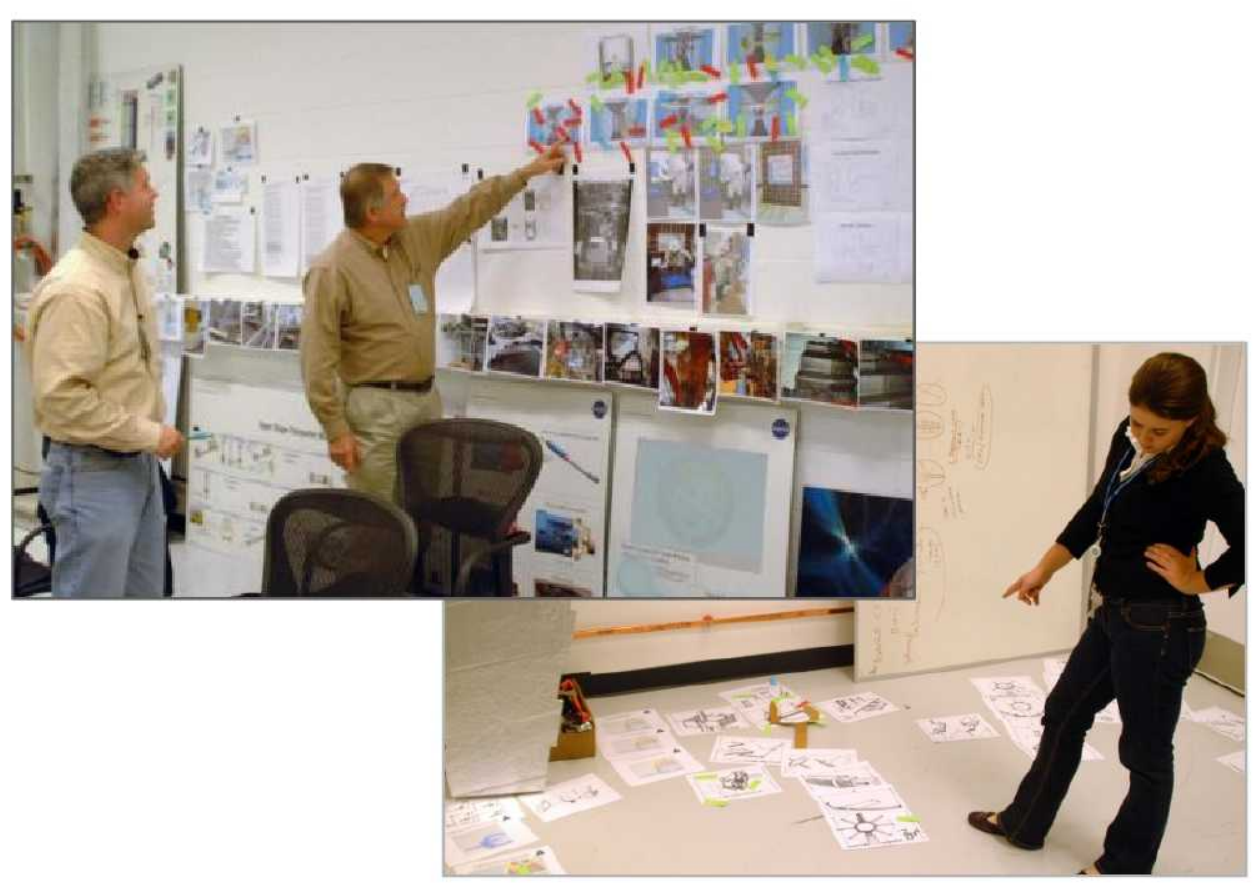




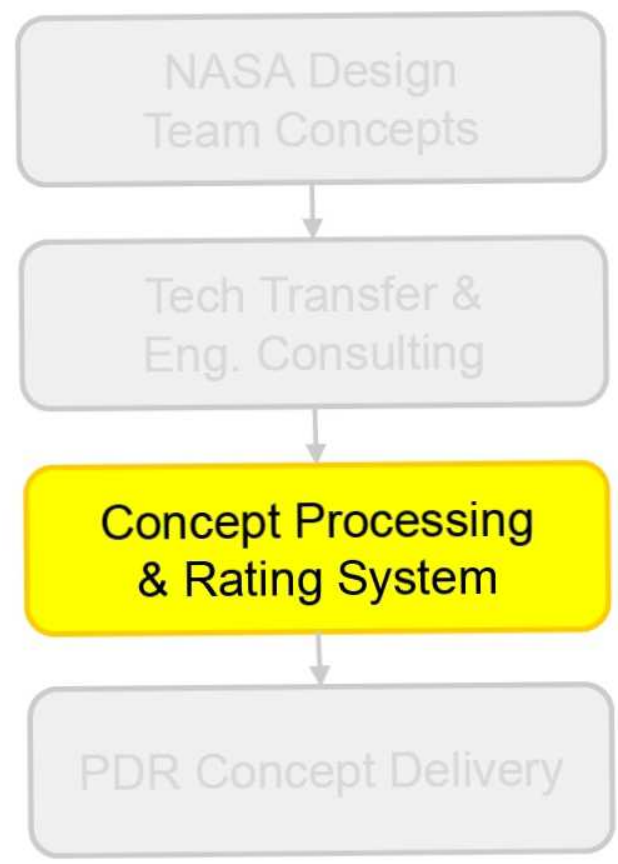

\section{Systems Engineering > Team Sessions for Concept Organizing \& Grouping (cont.)}

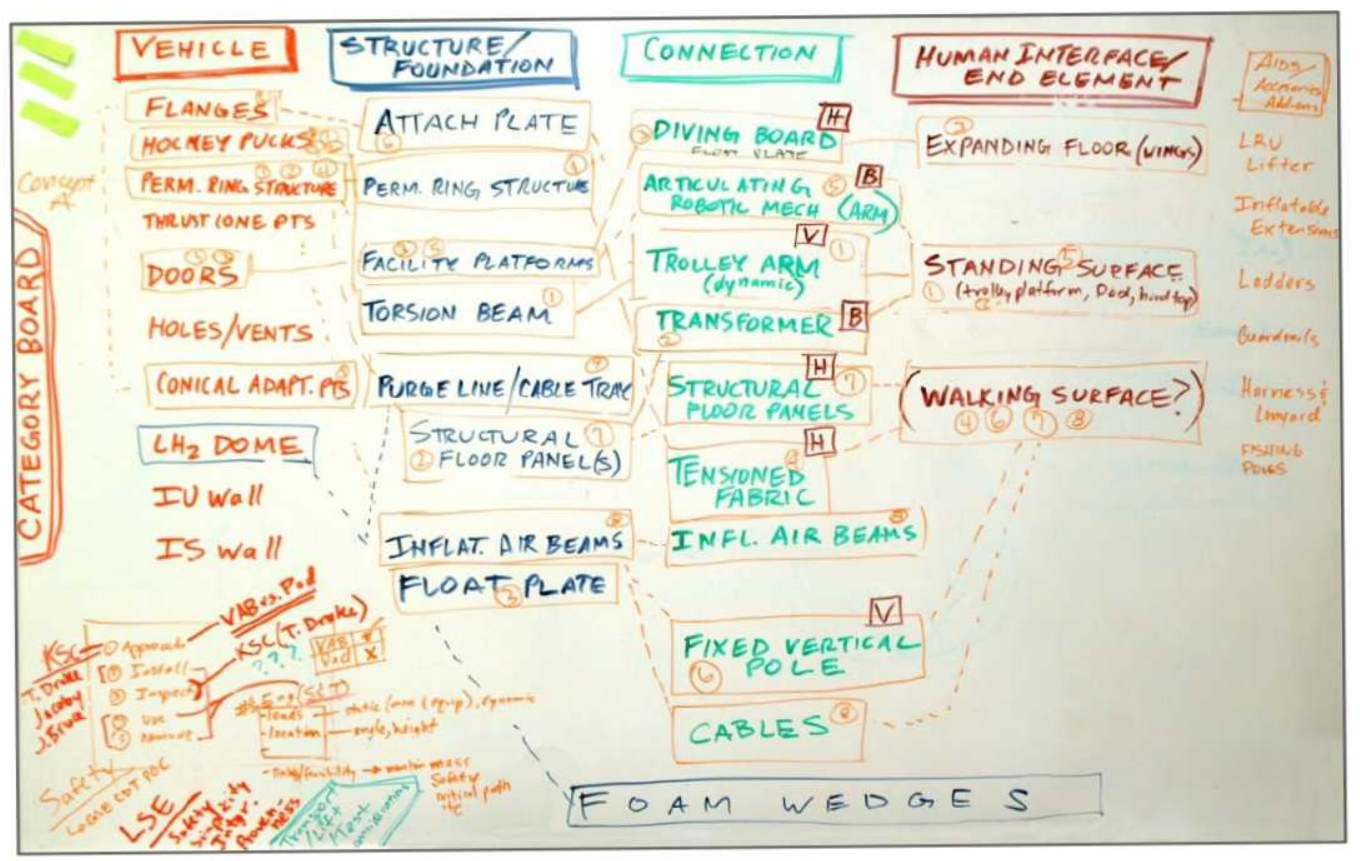




\section{CONCEPT DEVELOPMENT}

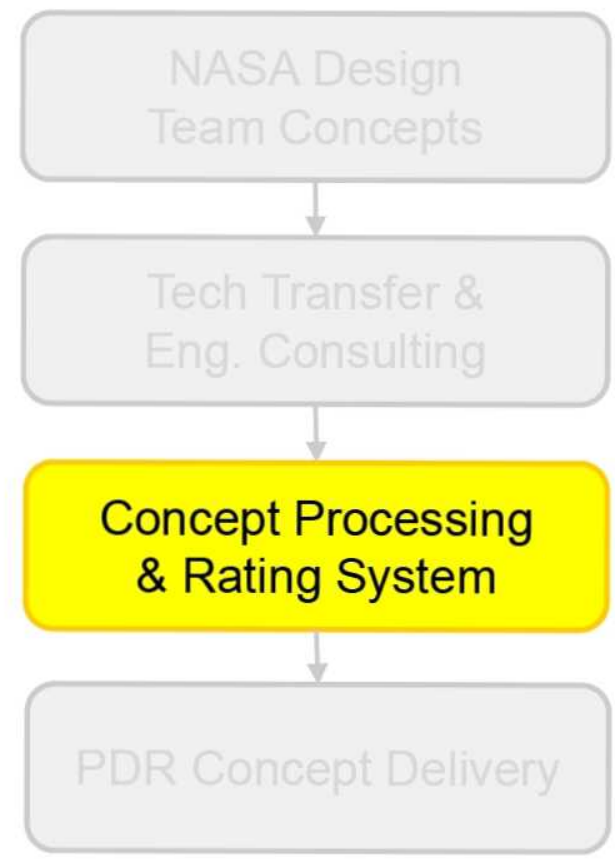

\section{Systems Engineering > Requirements-Based}

Criteria \& Rating Sessions

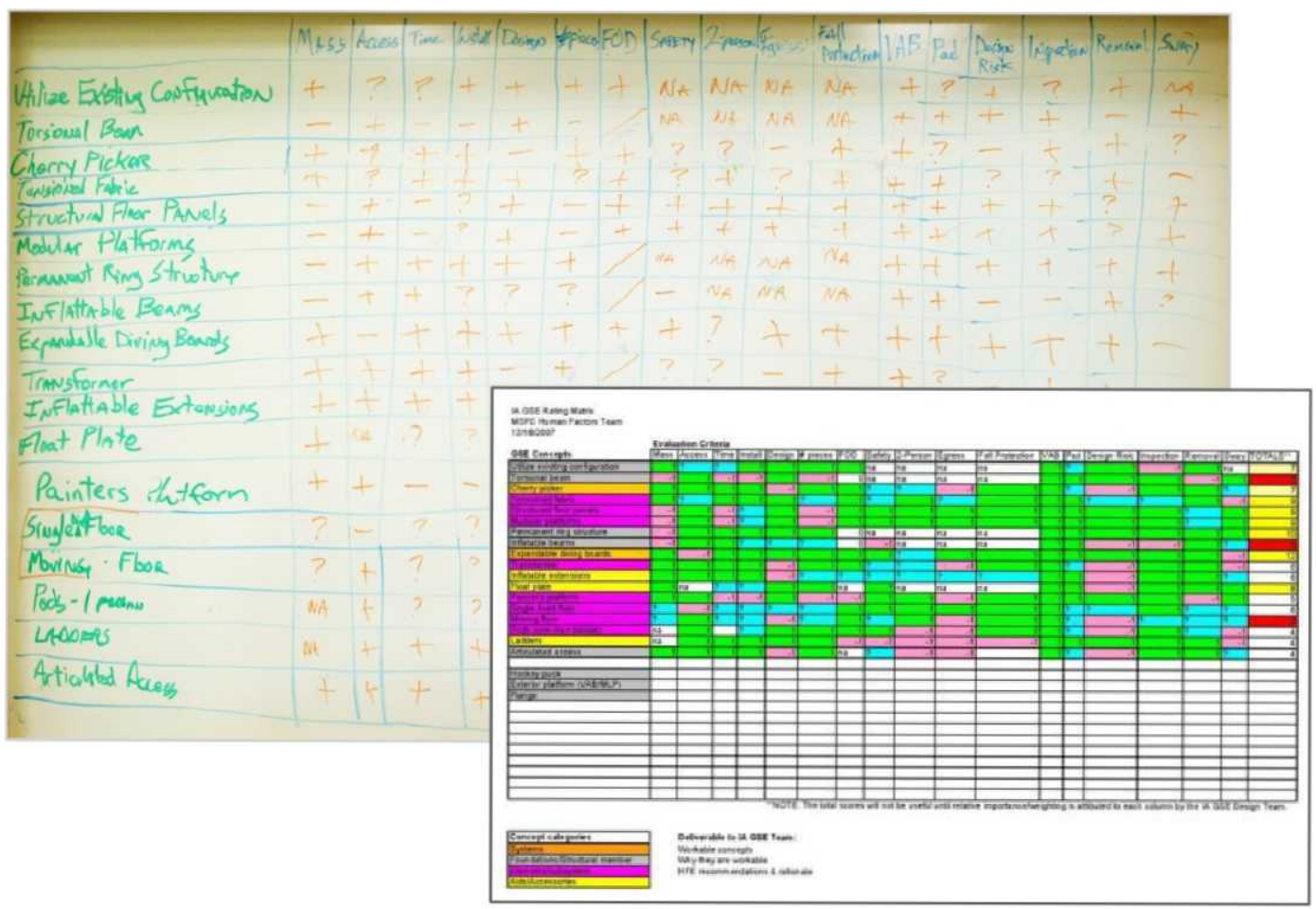




\section{CONCEPT DEVELOPMENT}

\section{Systems Engineering > Initial Selected Concepts}
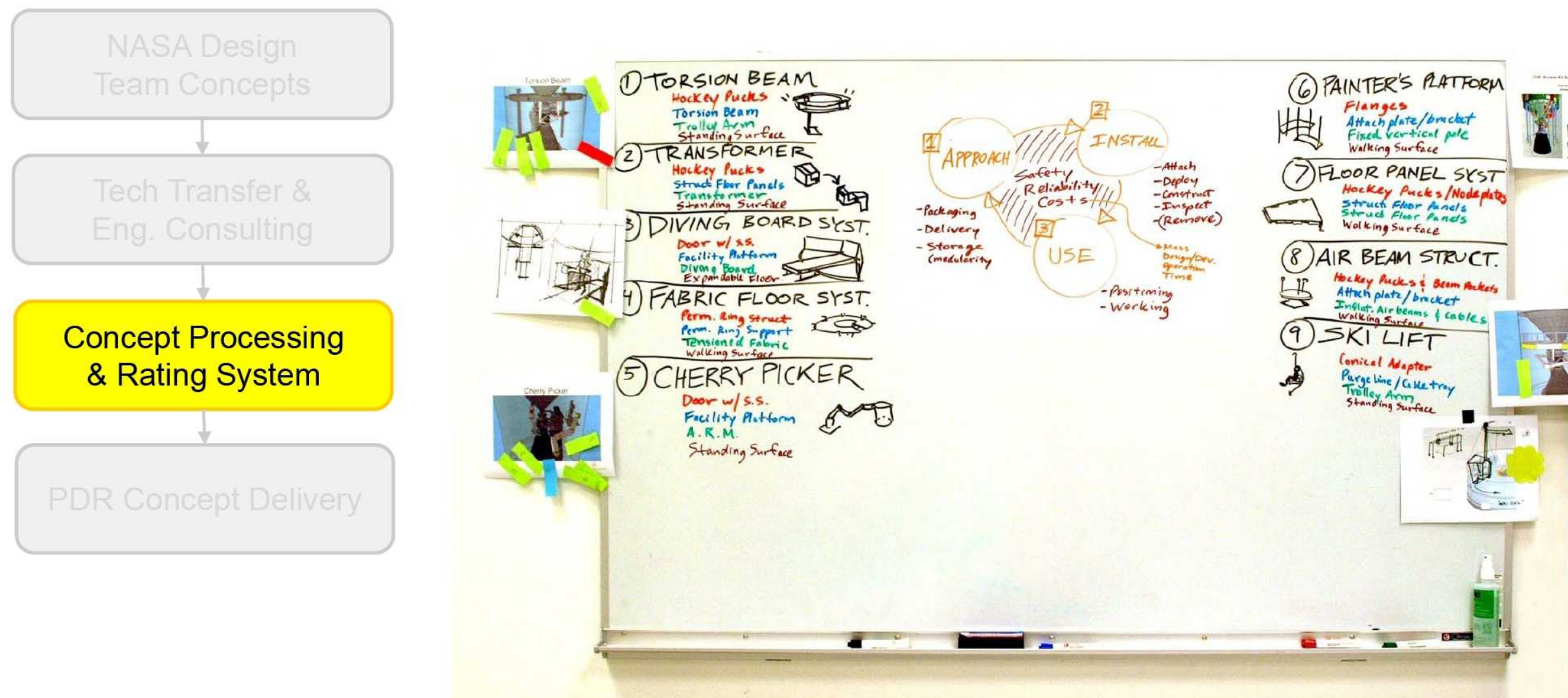


\section{Systems Engineering > Program Impacts}
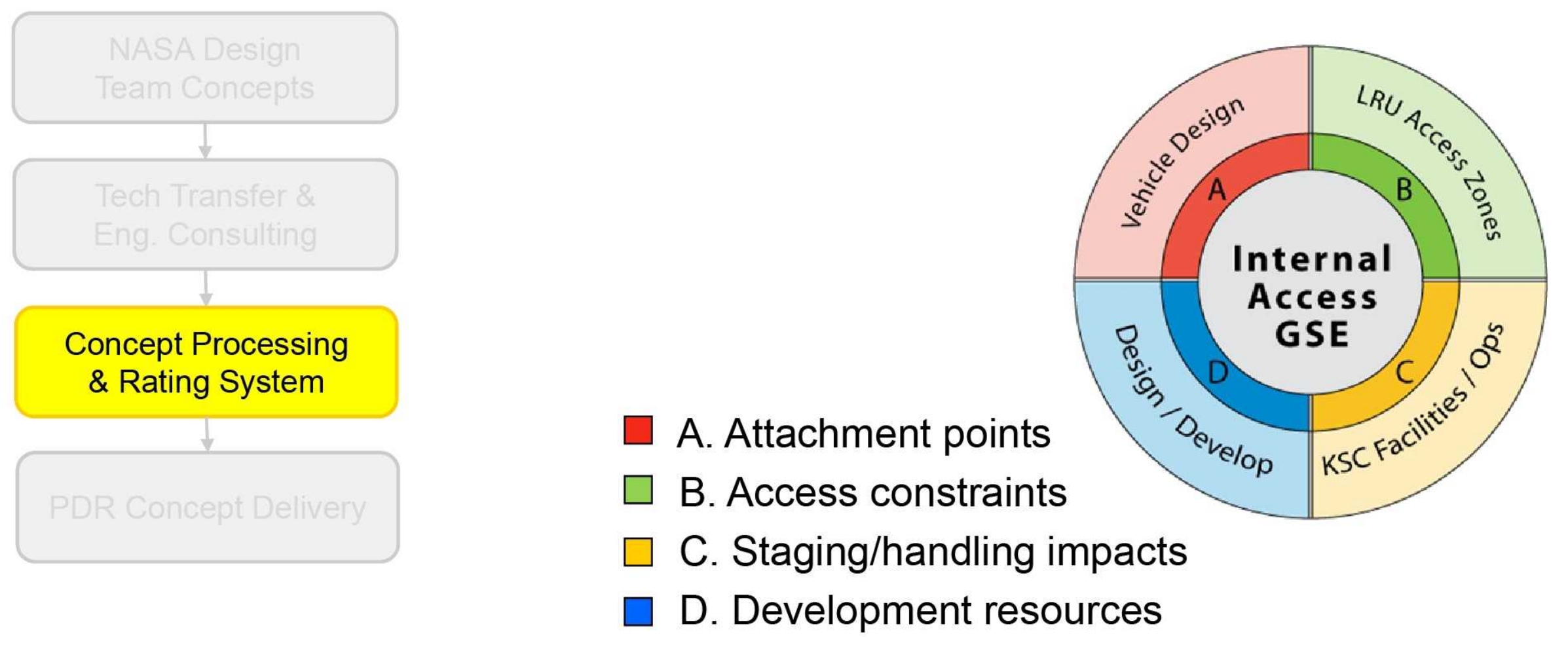


\section{CONCEPT DEVELOPMENT}

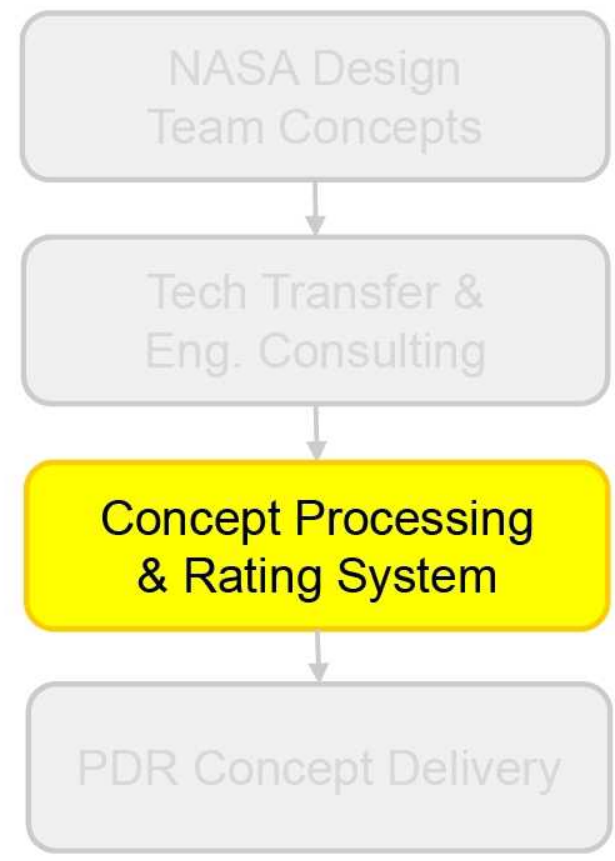

\section{Systems Engineering $>$ Refined Concepts for Stakeholder Selection Phase}

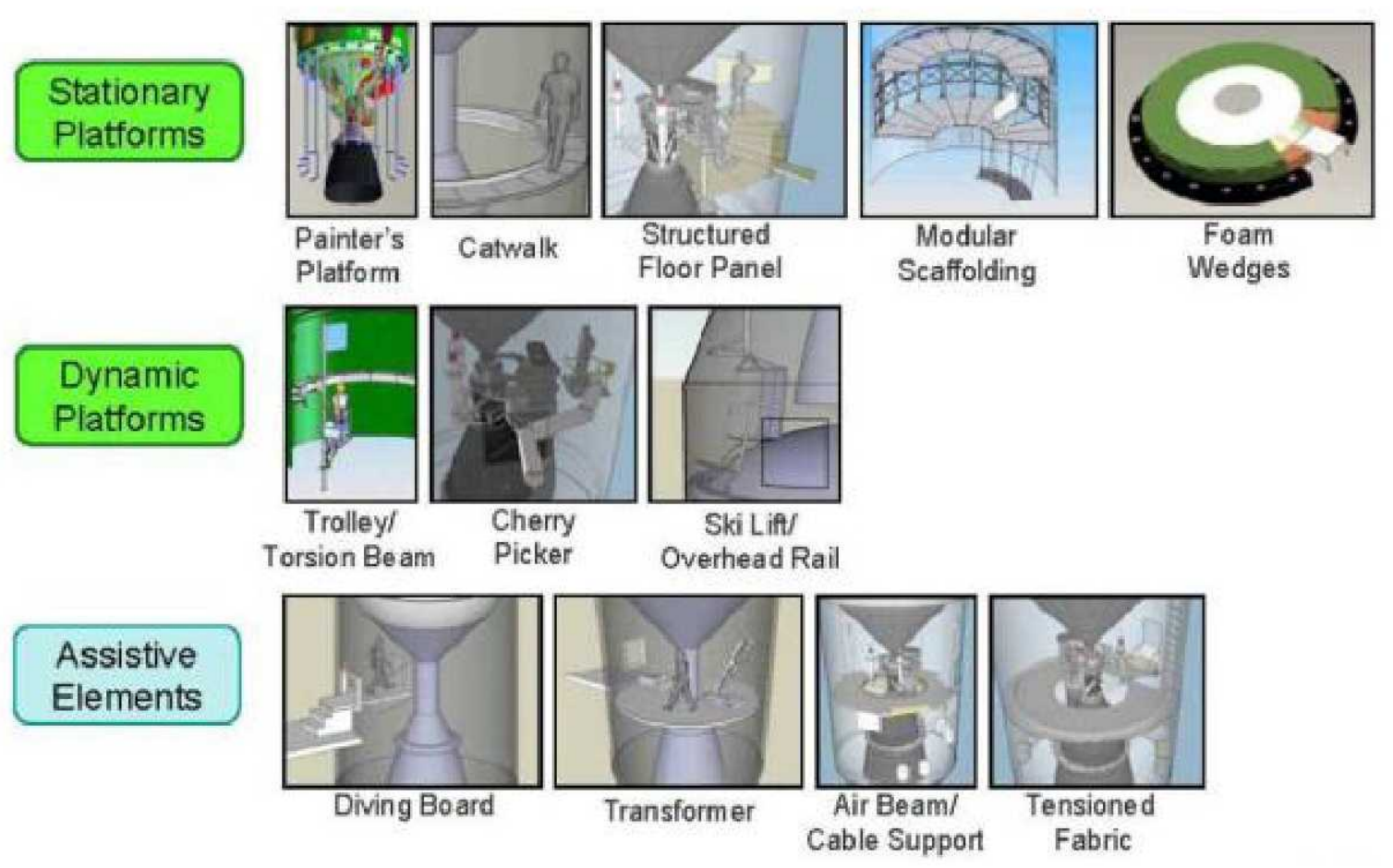




\section{CONCEPT DEVELOPMENT}

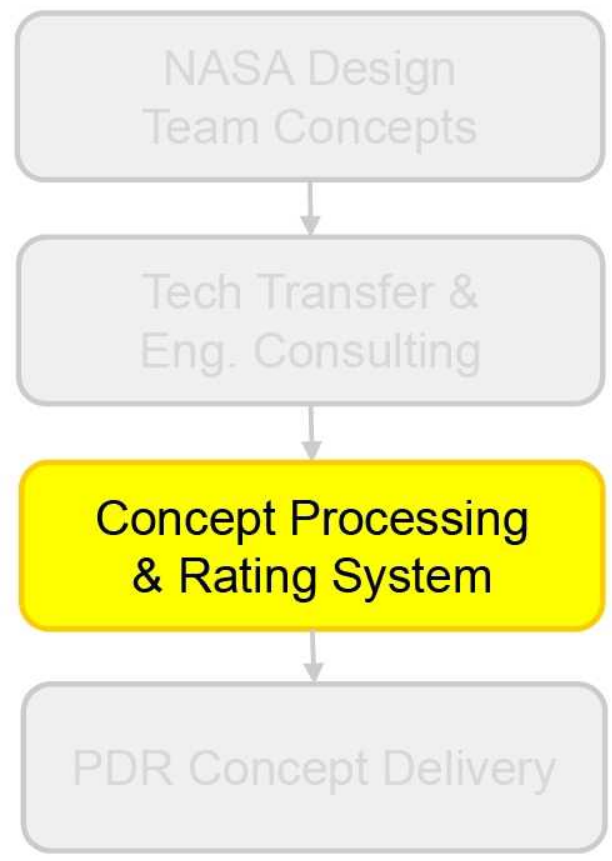

\section{Systems Engineering $>$ Refined Concepts for Stakeholder Selection Phase}

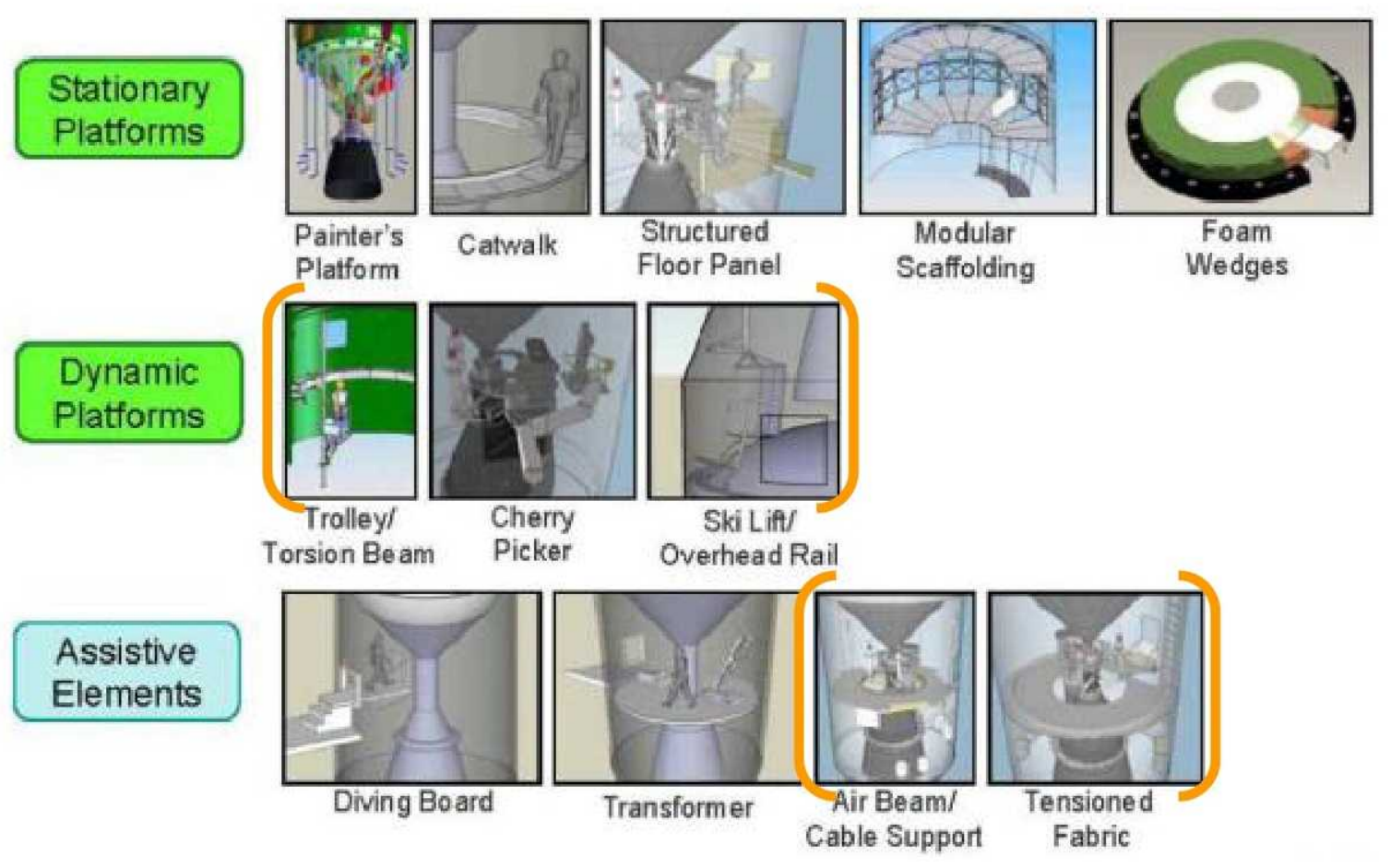




\section{CONCEPT DEVELOPMENT}

\section{Systems Engineering > Stakeholder Rating}
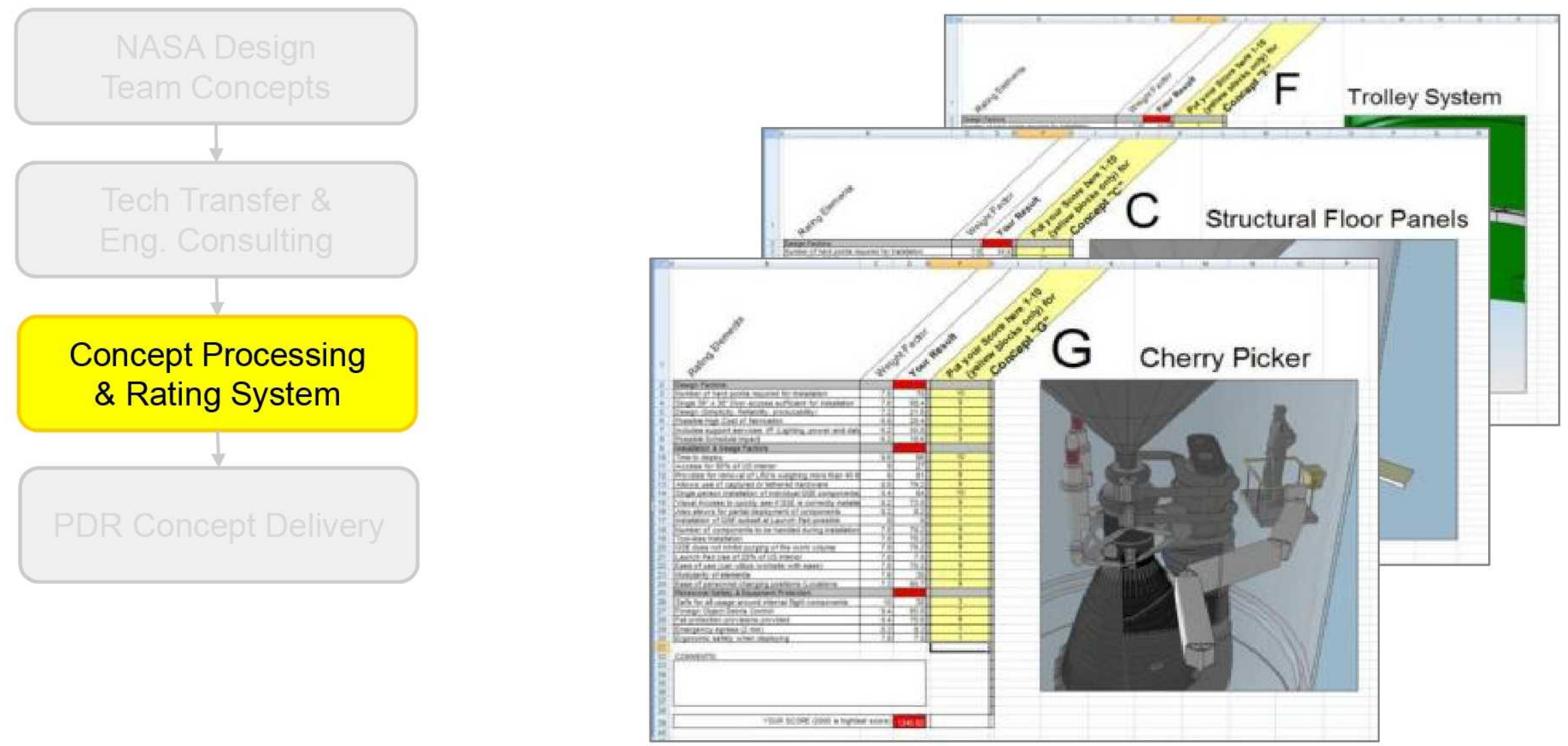


\section{CONCEPT DEVELOPMENT}

\section{Systems Engineering > Final Rating Results}
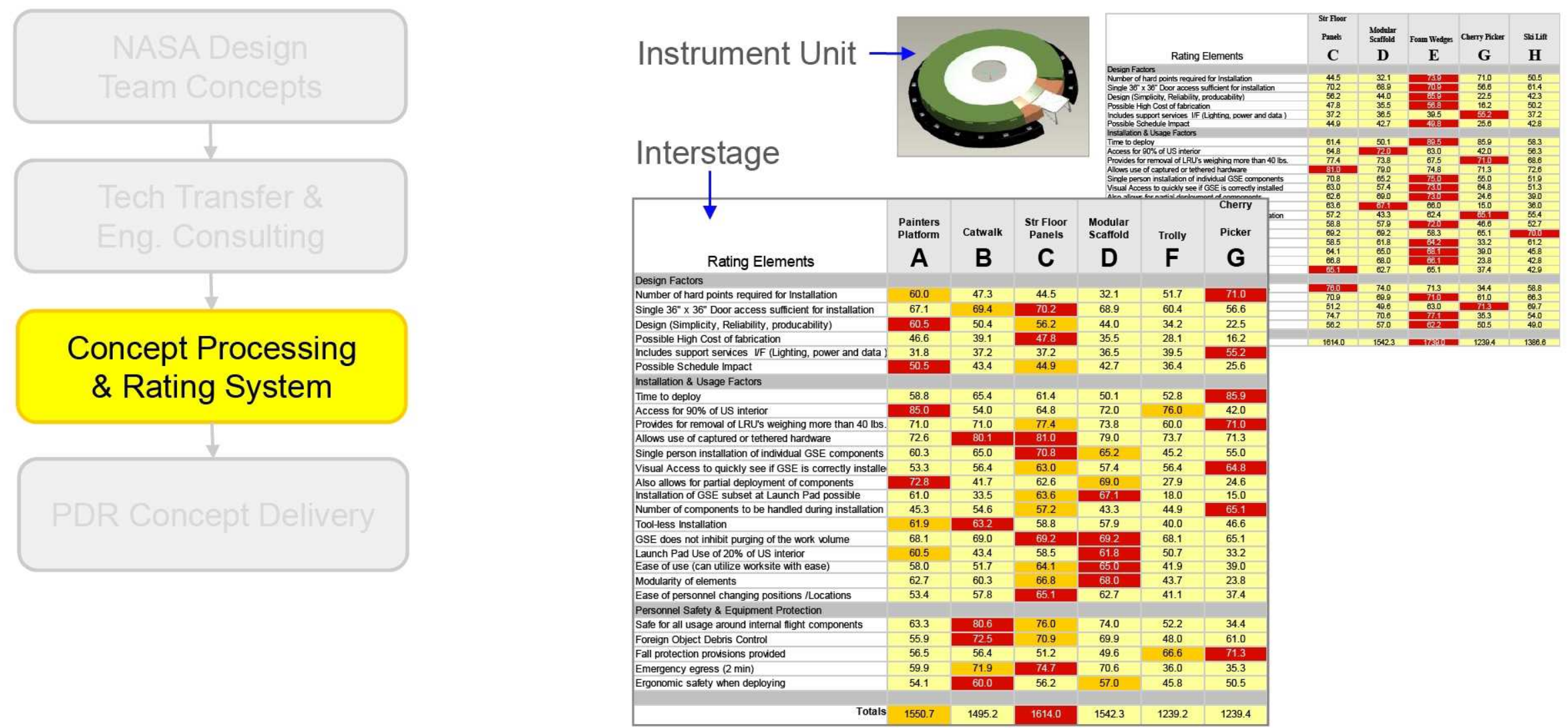


\section{CONCEPT DEVELOPMENT}

\section{Systems Engineering > Combined Concepts}
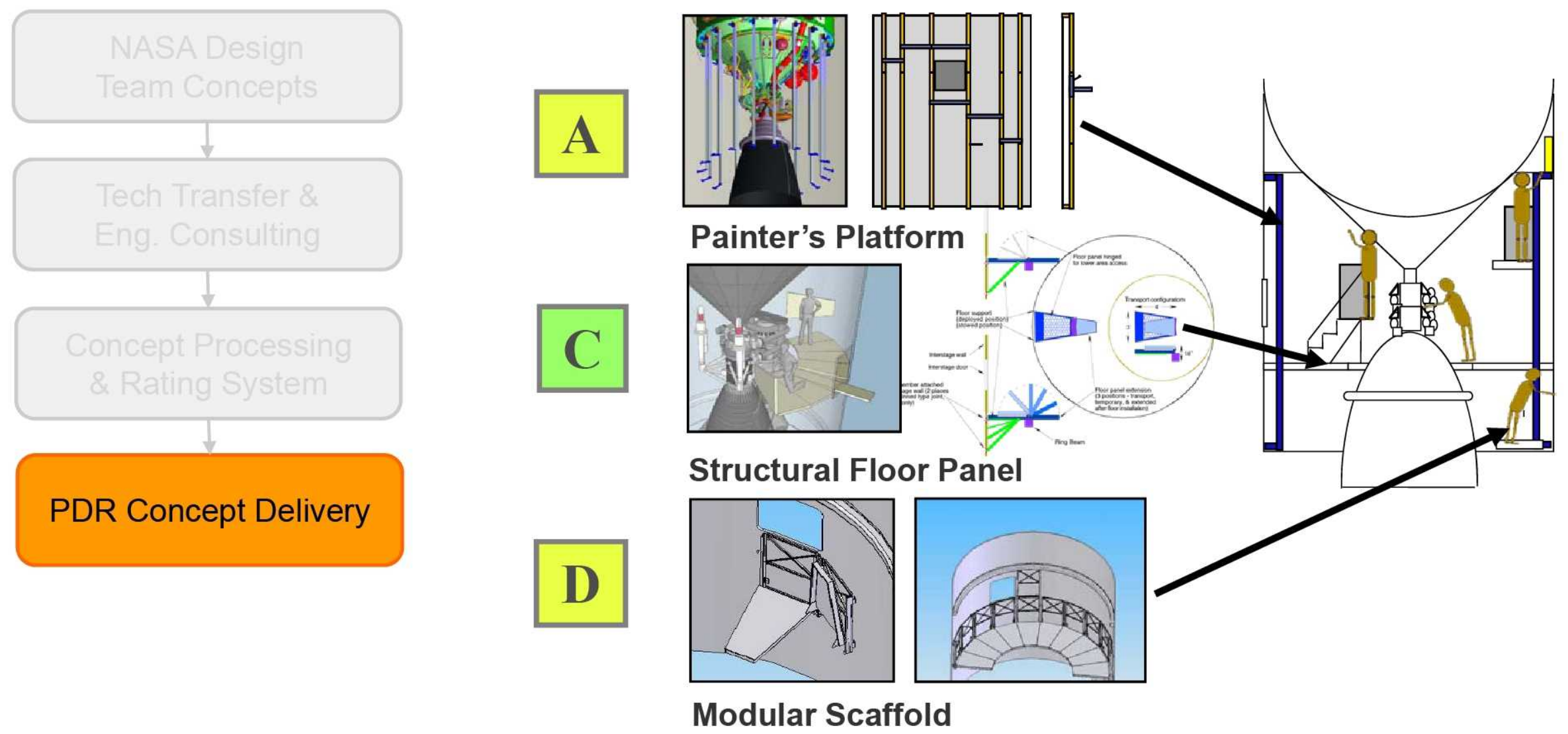


\section{AGENDA}

Design Opportunity

- Problem statement

- Requirements \& constraints

Concept Development

" User-centered design

- Technology transfer

" Systems engineering

\section{Results \& Discussion}

- Final concepts

- Challenges \& successes

- Lessons learned 


\section{RESULTS \& DISCUSSION}

\section{Final Concepts > Instrument Unit:}
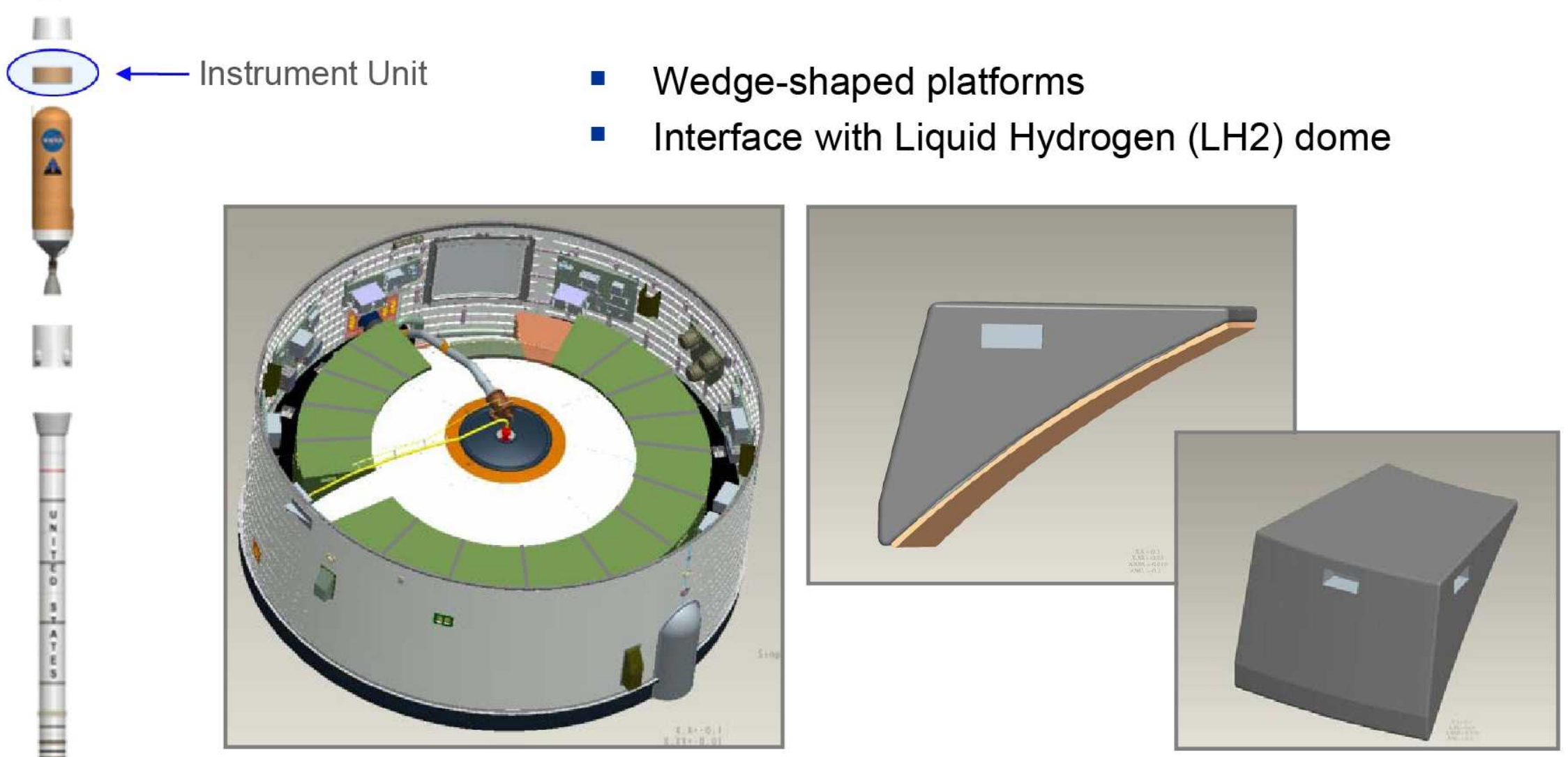


\section{RESULTS \& DISCUSSION}

\section{Final Concepts > Instrument Unit (cont.):}
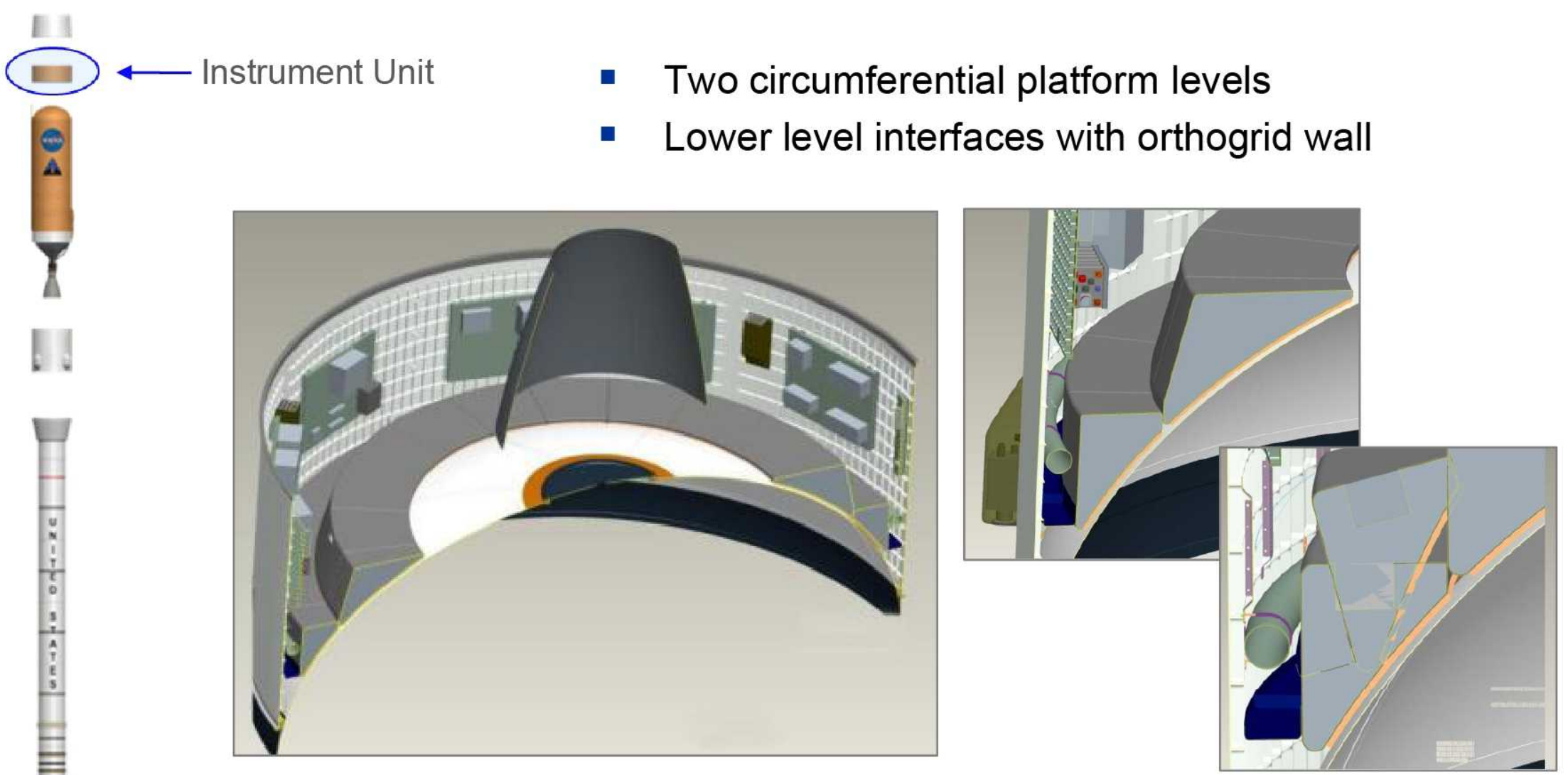


\section{RESULTS \& DISCUSSION}

\section{Final Concepts > Instrument Unit (cont.):}

$\longleftarrow$ Instrument Unit

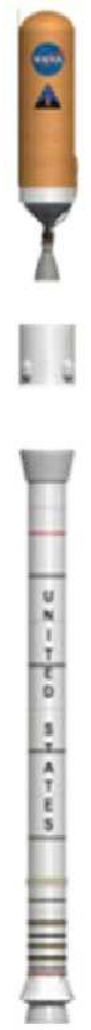

- Top level provides walking \& sitting surface

- Front face angled to increase operator clearance
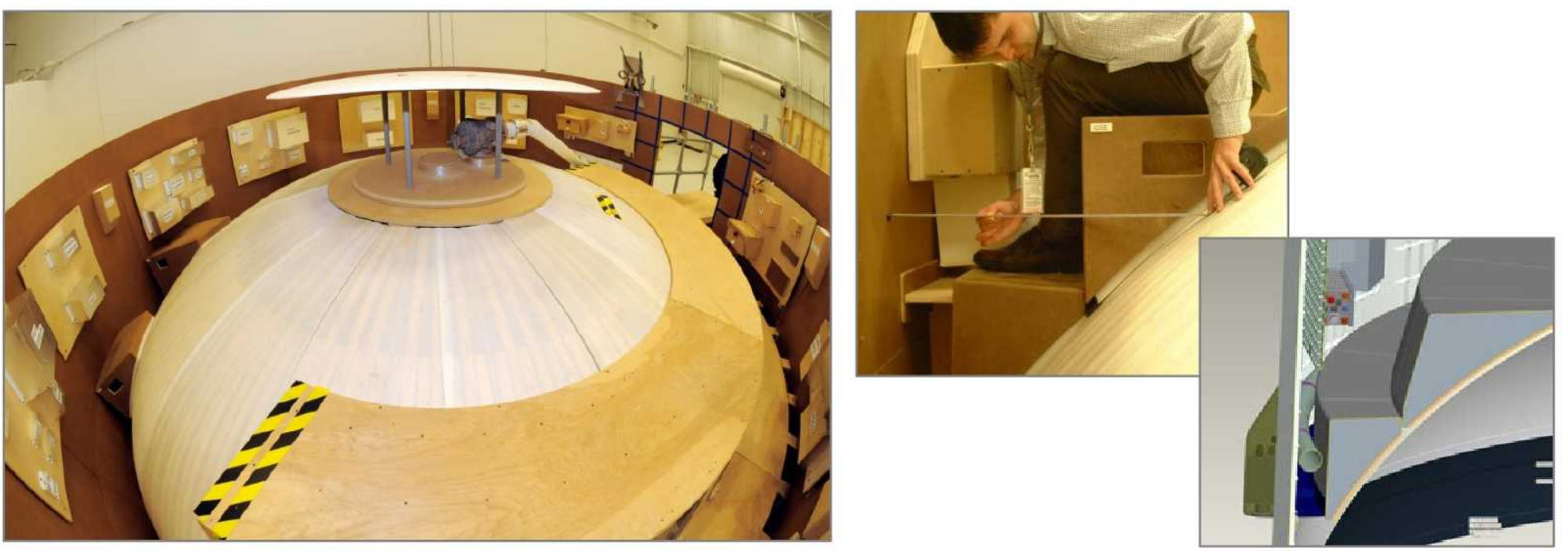


\section{RESULTS \& DISCUSSION}

\section{Final Concepts > Interstage:}

- Structural floor panels \& vertical supports

- Require permanent attachment points

\section{Interstage}
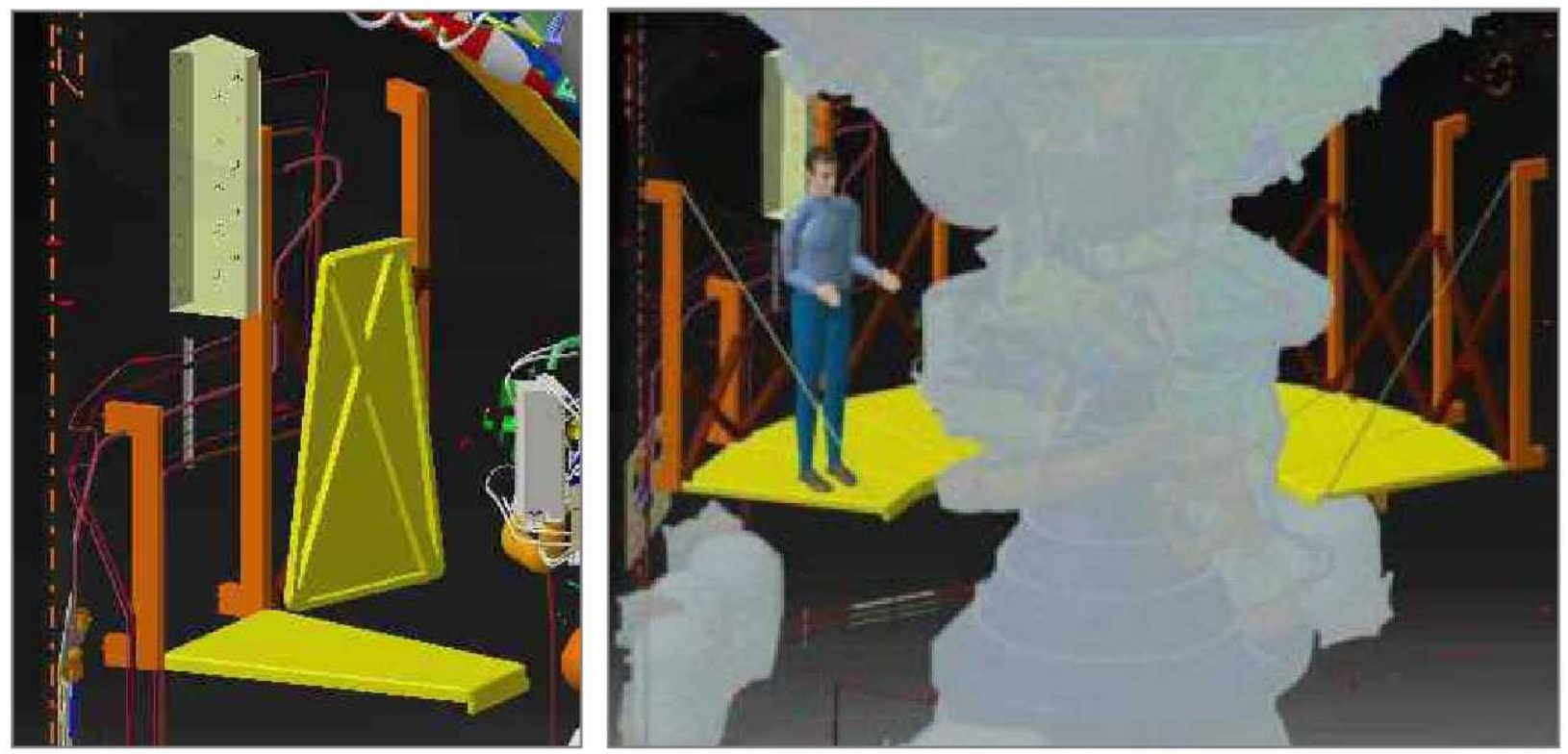


\section{RESULTS \& DISCUSSION}

\section{Final Concepts > Interstage (cont.):}
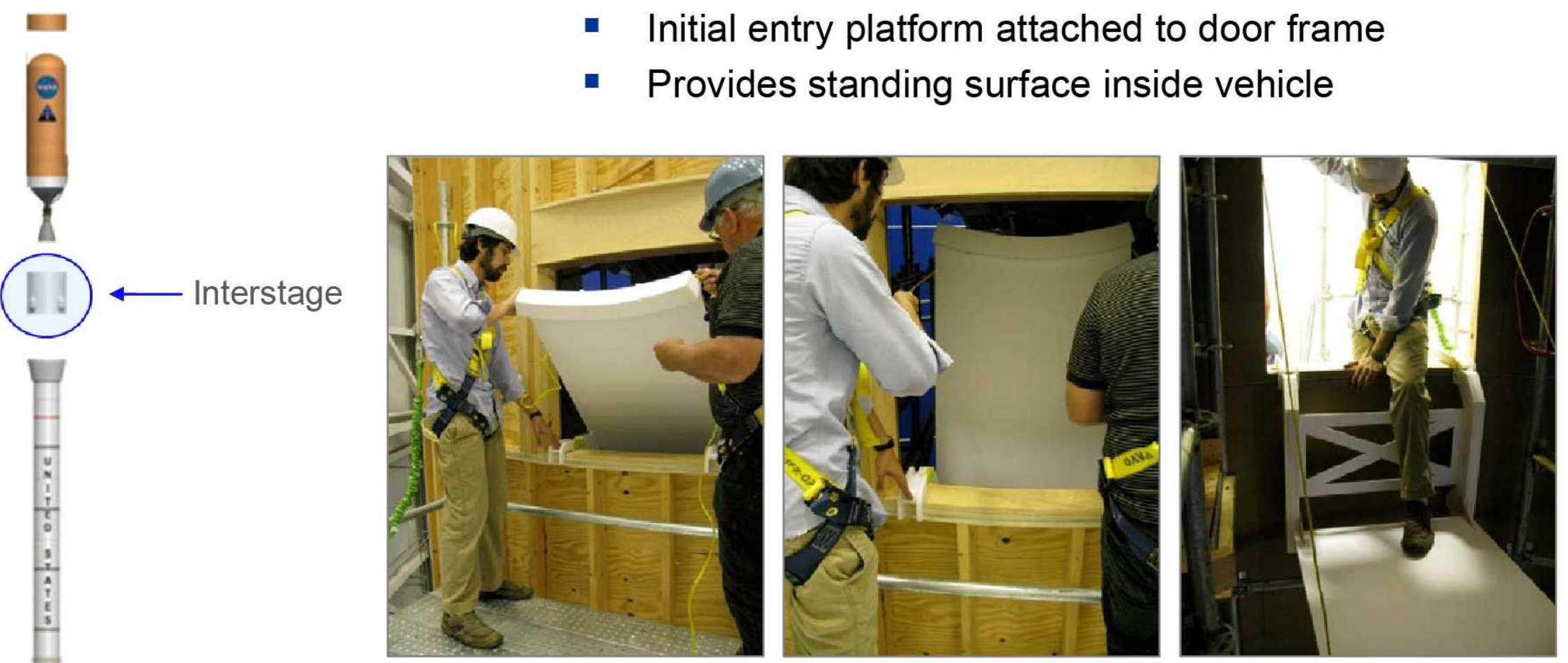


\section{RESULTS \& DISCUSSION}

\section{Final Concepts > Interstage (cont.):}

.

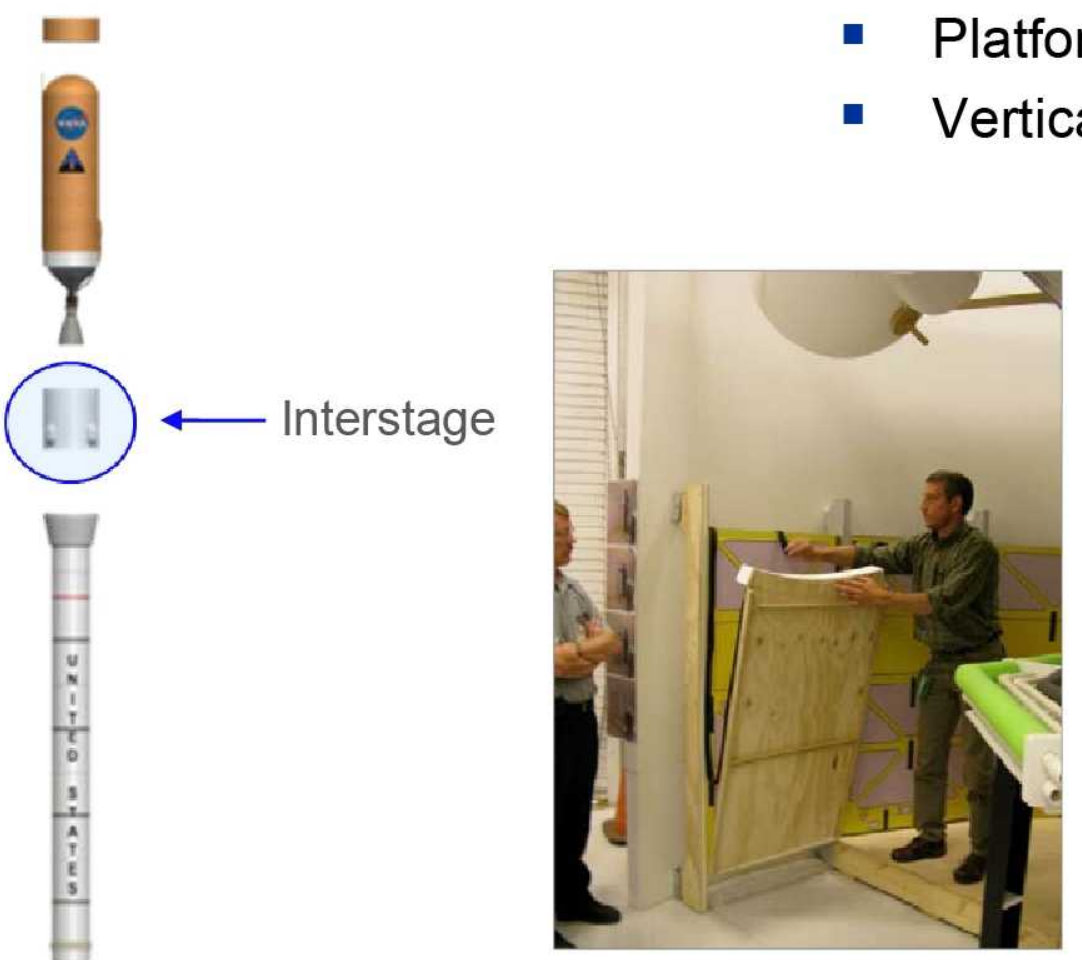

- Vertical \& horizontal hinges enable safe installation
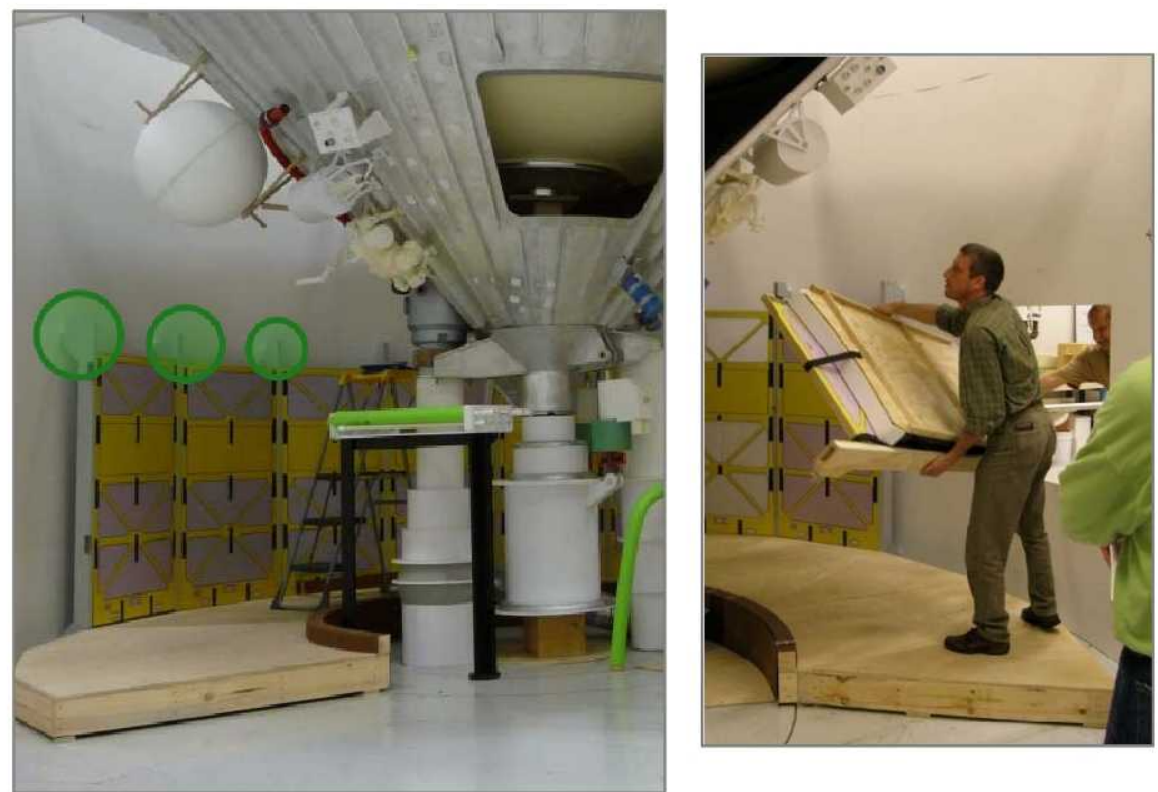


\section{RESULTS \& DISCUSSION}

\section{Challenges:}

- New technologies \& concepts

- Development costs / timelines

- Requirements compliance

- Safety concerns
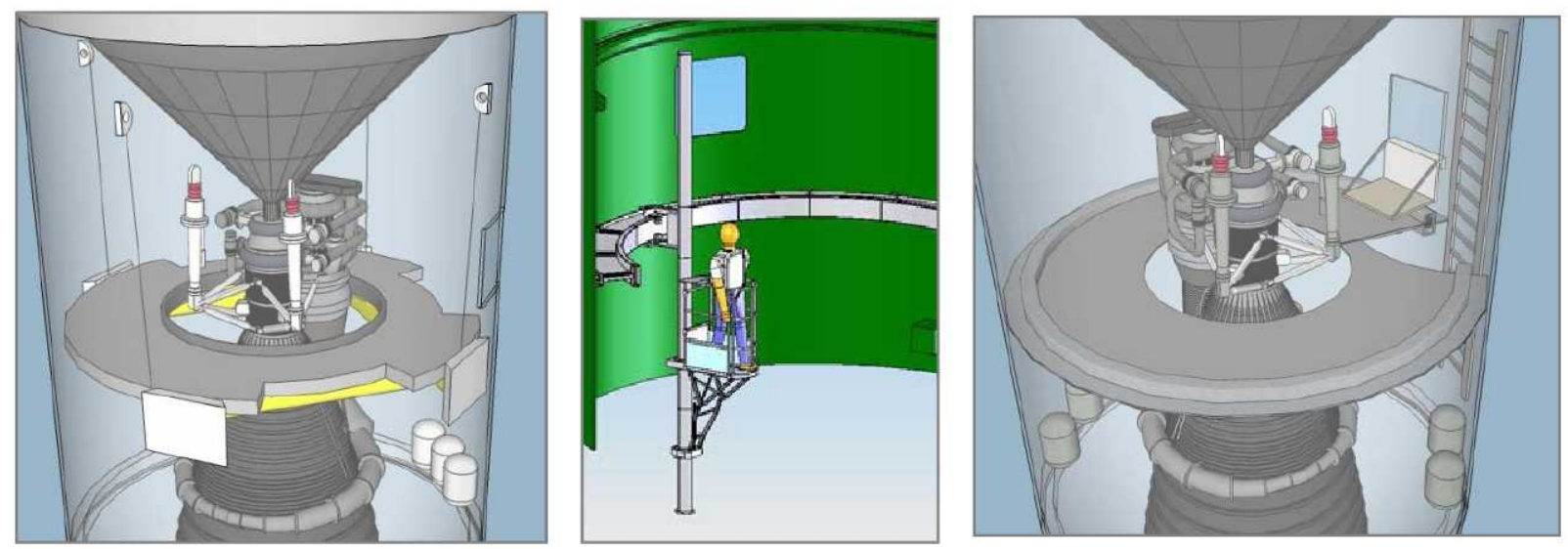


\section{RESULTS \& DISCUSSION}

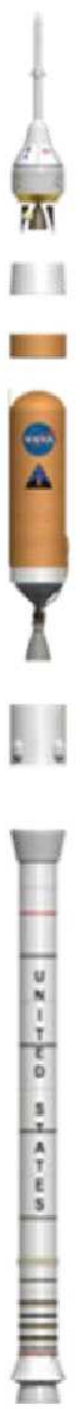

\section{Challenges:}

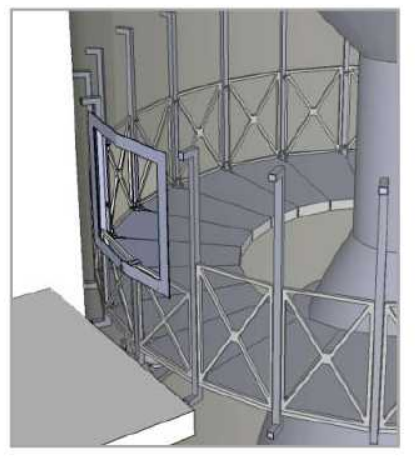

- New technologies \& concepts

- Development costs / timelines

- Requirements compliance

- Safety concerns

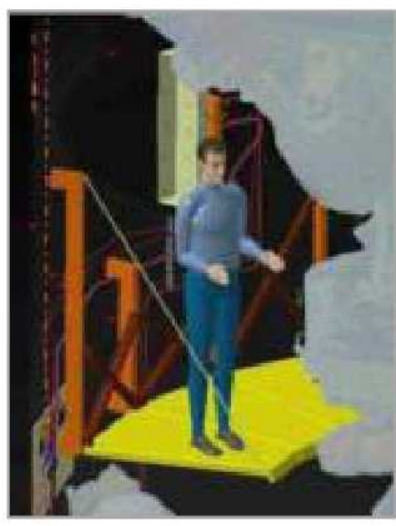

- Computer Aided Design (CAD)

- Rapid visualization vs. accurate models

- Appropriate method for deliverable 


\section{RESULTS \& DISCUSSION}

\section{Challenges:}

- New technologies \& concepts

- Development costs / timelines

- Requirements compliance

- Safety concerns

- Computer Aided Design (CAD)

- Rapid visualization vs. accurate models

- Appropriate method for deliverable

- Engineering consulting

- Partnership scope

- Concept influence 


\section{RESULTS \& DISCUSSION}

\section{Successes:}

- Internal Access GSE interfaces delivered for PDR

- Attachment points

- Mass allocations

- Keep-out zones
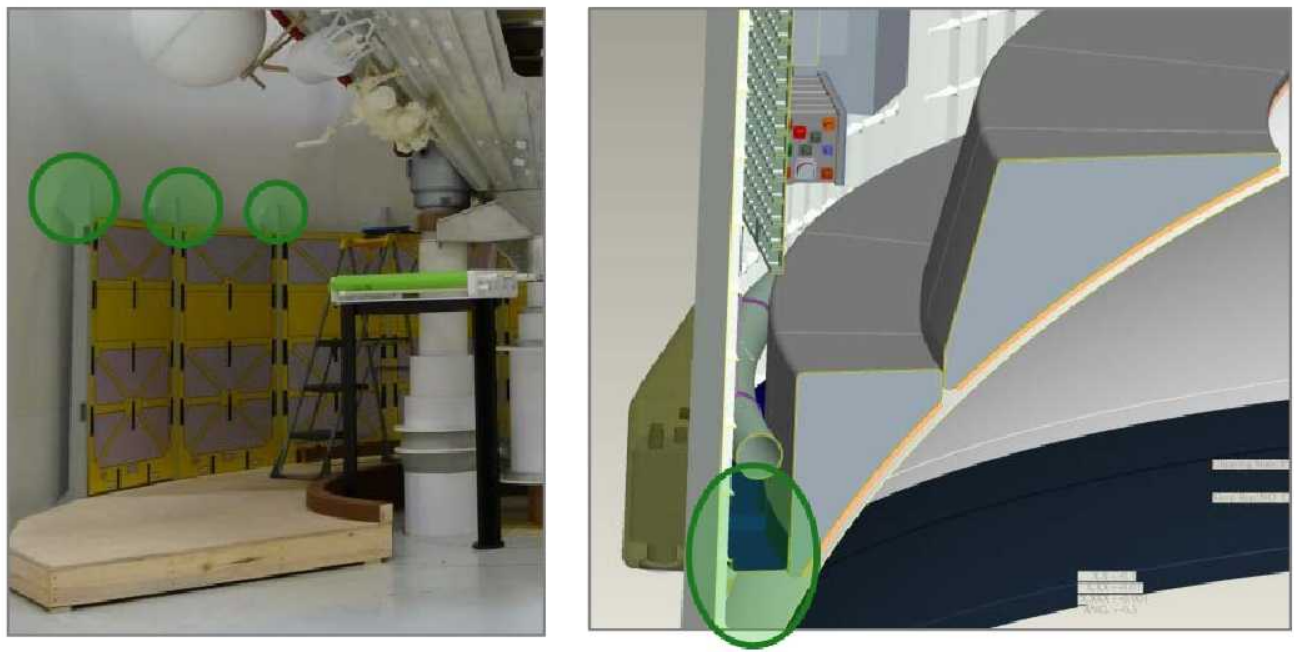


\section{RESULTS \& DISCUSSION}

\section{Successes:}

- Internal Access GSE interfaces delivered for PDR

- Attachment points

- Mass allocations

- Keep-out zones

- Requirement clarification (LH2 tank) 


\section{RESULTS \& DISCUSSION}

\section{Successes:}

- Internal Access GSE interfaces delivered for PDR

- Attachment points

- Mass allocations

- Keep-out zones

- Requirement clarification (LH2 tank)

- Increased awareness of Human Factors

- Operator tasks

- User-centered process

- Integrated design 


\section{RESULTS \& DISCUSSION}

\section{Lessons Learned:}

- Focus on vehicle \& human safety

- Minimize impacts

- Mass

- Time

- Cost 


\section{RESULTS \& DISCUSSION}

\section{Lessons Learned:}

- Focus on vehicle \& human safety

- Minimize impacts

- Mass

- Time

- Cost

- Know requirements \& rationale 


\section{RESULTS \& DISCUSSION}

\section{Lessons Learned:}

- Focus on vehicle \& human safety

- Minimize impacts

Mass

- Time

- Cost

- Know requirements \& rationale

- Visualization matters

- $\quad$ Sketch = discussion

- $\mathrm{CAD}=$ development 


\section{RESULTS \& DISCUSSION}

\section{Lessons Learned:}

- Focus on vehicle \& human safety

- Minimize impacts

- Mass

- Time

- Cost

* Know requirements \& rationale

- Visualization matters

- Sketch $=$ discussion

- $\mathrm{CAD}=$ development

- Encourage conflict \& opposing views 


\section{RESULTS \& DISCUSSION}

\section{Lessons Learned:}

- Focus on vehicle \& human safety

- Minimize impacts

Mass

- Time

Cost

- Know requirements \& rationale

- Visualization matters

- Sketch $=$ discussion

- $\mathrm{CAD}=$ development

- Encourage conflict \& opposing views

- Maintain creative processes

- Management support

- Industry connections 


\section{CONCLUSIONS \& FUTURE WORK}

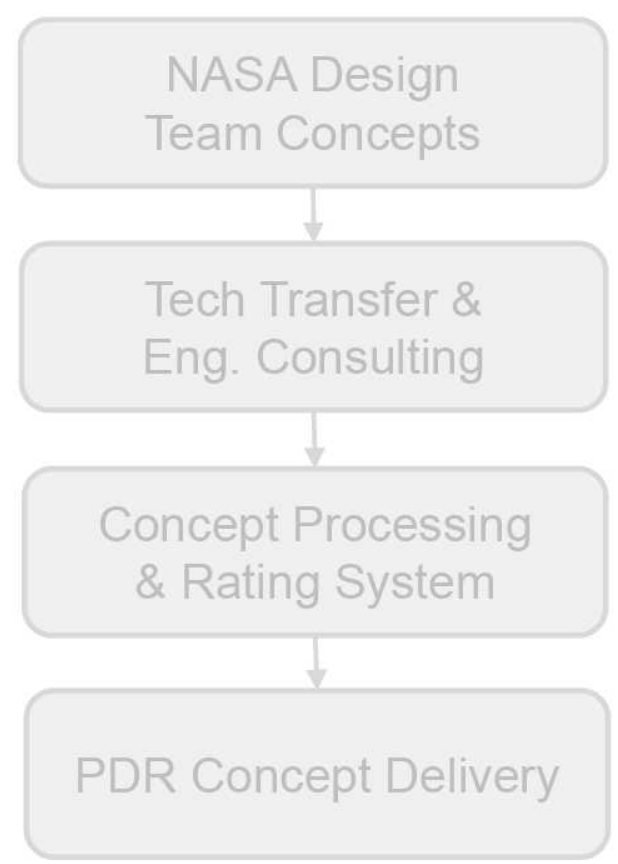

- NASA culture challenges unproven technology

- Present credible concepts $\mathrm{w} /$ requirements-focus 


\section{CONCLUSIONS \& FUTURE WORK}

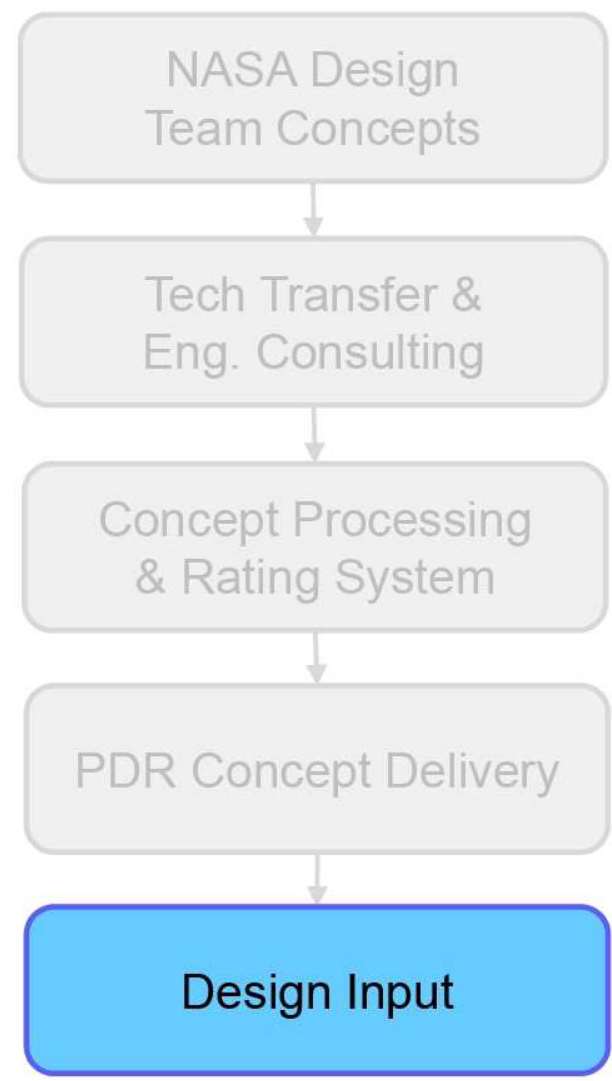

- NASA culture challenges unproven technology

- Present credible concepts w/ requirements-focus

- Integrated system design

- Operator shadowing

- Industry research

- Human interfaces

- Worksite equipment for tasks

- Vehicle interior configuration

- Design development with subsystems 


\section{CONCLUSIONS \& FUTURE WORK}

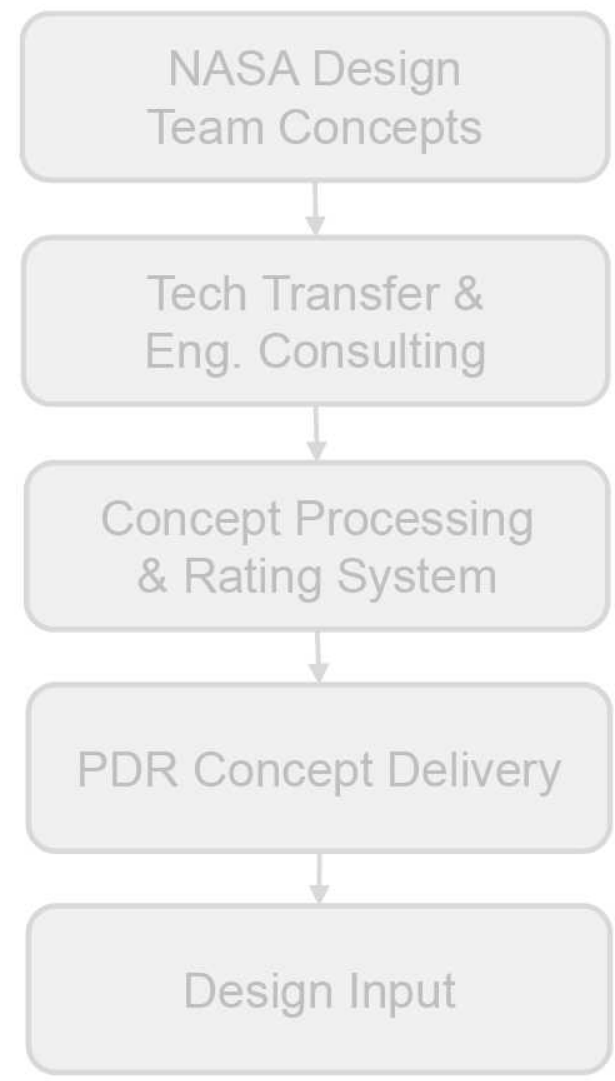

- NASA culture challenges unproven technology

- Present credible concepts w/ requirements-focus

- Integrated system design

- Operator shadowing

- Industry research

- Human interfaces

II Worksite equipment for tasks

- Vehicle interior configuration

- Design development with subsystems

- Improve Technology Transfer relationship

- Ares V

- Altair

- Spin-off 


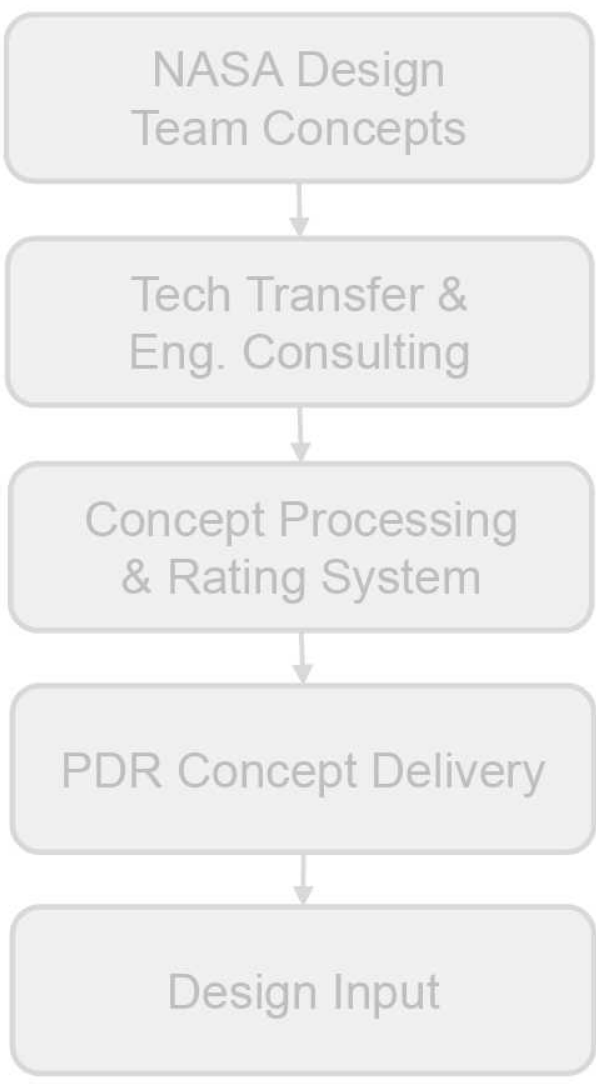

Jason Quick, Human Factors Engineer Triumph Aerospace System - Newport News Marshall Space Flight Center, Huntsville, AL jason.c.quick@nasa.gov 256-544-7158
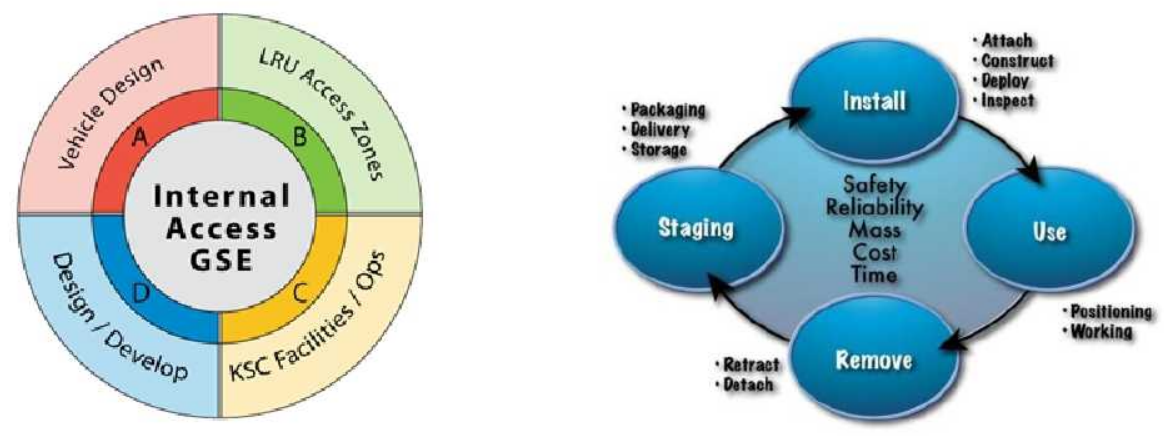

\section{Technology Transfer Challenges: A Case Study of User-Centered Design in NASA's Systems Engineering Culture}

